\title{
STRUCTURE OF $\operatorname{Gal}\left(\mathbb{k}_{2}^{(2)} / \mathbb{k}\right)$ FOR SOME FIELDS $\mathbb{k}=\mathbb{Q}\left(\sqrt{2 p_{1} p_{2}}, i\right)$ WITH $\mathbf{C l}_{2}(\mathbb{k}) \simeq(2,2,2)$
}

\author{
ABDELMALEK AZIZI, ABDELKADER ZEKHNINI, AND MOHAMMED TAOUS
}

\begin{abstract}
Let $p_{1} \equiv p_{2} \equiv 5(\bmod 8)$ be different primes. Put $i=\sqrt{-1}$ and $d=2 p_{1} p_{2}$, then the bicyclic biquadratic field $\mathrm{k}=\mathbb{Q}(\sqrt{d}, i)$ has an elementary abelian 2-class group of rank 3. In this paper we determine the nilpotency class, the coclass, the generators and the structure of the non-abelian Galois group $\operatorname{Gal}\left(\mathbb{k}_{2}^{(2)} / \mathbb{k}\right)$ of the second Hilbert 2-class field $\mathbb{k}_{2}^{(2)}$ of $\mathbb{k}$. We study the capitulation problem of the 2-classes of $k$ in its seven unramified quadratic extensions $\mathbb{K}_{i}$ and in its seven unramified bicyclic biquadratic extensions $\mathbb{L}_{i}$.
\end{abstract}

\section{INTRODUCTION}

Let $k$ be an algebraic number field and $C l_{2}(k)$ be its 2-class group i.e. the Sylow 2-subgroup of the ideal class group, $C l(k)$, of $k$. Denote by $k_{2}^{(1)}$ the Hilbert 2-class field of $k$ and by $k_{2}^{(2)}$ its second Hilbert 2-class field. Put $G=\operatorname{Gal}\left(k_{2}^{(2)} / k\right)$ and let $G^{\prime}$ denote its derived group, then it is well known that $C / G^{\prime} \simeq C l_{2}(k)$. The knowledge of $G$, its structure and its generators solves a lot of problems in number theory as capitulation problems, the finiteness or not of the towers of number fields and the structures of the 2-class groups of the unramified extensions of $k$ within $k_{2}^{(1)}$. For particular types of fields $\mathrm{k}$, for example, fields with $C l_{2}(k) \simeq(2,2)$, the structure of $G$ has been completely determined (see [13]). The success in this case is in part due to the fact that, contrary to most other cases, 2-groups whose abelianization is $(2,2)$ are well understood, cf. [15] and [14].

If one considers another case, namely where $k=\mathbb{Q}(\sqrt{d}, i)$ and $C l_{2}(k) \simeq(2,2,2)$, for some square-free integer $d$, then the situation is very different and very difficult when compared with the case described above; moreover there is no known way (to our knowledge) to determine the structure of $G$. Our aim in the present paper is to determine the isomorphism types of the second 2-class group of certain number

2010 Mathematics Subject Classification. 11R11, 11R29, 11R32, 11R37.

Key words and phrases. Galois group, Coclass, Class group, Capitulation, Hilbert class. 
fields $k=\mathbb{Q}(\sqrt{d}, i)$, to give the structure and generators of $G$ and we will explicitly determine ker $j_{K / k}$, the kernel of the natural class extension homomorphism $j_{K / k}$ : $C l(k) \longrightarrow C l(K)$, where $K$ is an unramified extension of $k$ within $k_{2}^{(1)}$. It should be noted that the determination of ker $j_{K / k}$ is not always easy to do, especially when $K=k(\sqrt{a+b \sqrt{-1}})$ or $K=k\left(\sqrt{a+b \sqrt{-1}}, \sqrt{a^{\prime}+b^{\prime} \sqrt{-1}}\right)$, with some positive integers $a, b, a^{\prime}$ and $b^{\prime}$.

Let $m$ be a square-free integer and $K$ be a number field. Throughout this paper, we adopt the following notations:

- $h(m),($ resp. $h(K))$ : the 2-class number of $\mathbb{Q}(\sqrt{m})$ (resp. $K)$.

- $\mathcal{O}_{K}$ : the ring of integers of $K$.

- $E_{K}$ : the unit group of $\mathcal{O}_{K}$.

- $W_{K}$ : the group of roots of unity contained in $K$.

- $\omega_{K}$ : the order of $W_{K}$.

- $K^{+}$: the maximal real subfield of $K$, if it is a CM-field.

- $Q_{K}=\left[E_{K}: W_{K} E_{K^{+}}\right]$is Hasse's unit index, if $K$ is a CM-field.

- $q(K / \mathbb{Q})=\left[E_{K}: \prod_{i}^{s} E_{k_{i}}\right]$ is the unit index of $K$, if $K$ is multiquadratic, where $k_{i}$ are the quadratic subfields of $K$.

- $K^{(*)}$ : the genus field of $K$.

- $\mathbf{C} l_{2}(K)$ : the 2-class group of $K$.

- $\varepsilon_{m}$ : the fundamental unit of $\mathbb{Q}(\sqrt{m})$.

- $i=\sqrt{-1}$.

- FSU: denotes a fundamental system of units.

\section{MAin RESUlts}

Let $p_{1} \equiv p_{2} \equiv 5(\bmod 8)$ be different primes, then there exist some positive integers $e, f, g$ and $h$ such that $p_{1}=e^{2}+4 f^{2}$ and $p_{2}=g^{2}+4 h^{2}$. Let $p_{1}=\pi_{1} \pi_{2}$ and $p_{2}=\pi_{3} \pi_{4}$, where $\pi_{1}=e+4 i f$ and $\pi_{2}=e-4 i f\left(\right.$ resp. $\pi_{3}=g+4 i h$ and $\left.\pi_{4}=g-4 i h\right)$ are conjugate prime elements in the cyclotomic field $k=\mathbb{Q}(i)$ dividing $p_{1}$ (resp. $p_{2}$ ). Denote by $\mathbb{k}$ the imaginary bicyclic biquadratic field $\mathbb{Q}(\sqrt{d}, i)$, where $d=2 p_{1} p_{2}$, its three quadratic subfields are $k=\mathbb{Q}(i), k_{0}=\mathbb{Q}(\sqrt{d})$ and $\bar{k}_{0}=\mathbb{Q}(\sqrt{-d})$. Let $\mathbb{k}_{2}^{(1)}$ be the Hilbert 2-class field of $\mathbb{k}, \mathbb{k}_{2}^{(2)}$ its second Hilbert 2-class field and $G$ be the Galois group of $\mathbb{k}_{2}^{(2)} / \mathbb{k}$. According to [5], $\mathbb{k}$ has an elementary abelian 2-class group $\mathrm{Cl}_{2}(\mathbb{k})$ of rank 3 , that is, of type $(2,2,2)$. In an earlier paper [4] we have proved that the 2-class field tower of $\mathrm{k}$ has length 2 , the order of $G$ is greater 
than or equal to 64 , we have given necessary and sufficient conditions to have $G$ of order 64 and we have shown that if $\mathbb{K}$ is an unramified quadratic extension of $\mathbb{k}$ other than $\mathbb{K}_{3}=\mathbb{k}(\sqrt{2})$, then $\mathbf{C l}_{2}(\mathbb{K})$ is of type $(2,4)$ or $(2,2,2)$. In this paper we complete this study by determining the structure of $G$, the abelian type invariants of the 2-class groups of all the unramified extensions of $\mathbb{k}_{\mathrm{k}}$ within $\mathrm{k}_{2}^{(1)}$ and the kernel of the natural class extension homomorphism $j_{\mathbb{K} / \mathbb{k}}: C l_{2}(\mathbb{k}) \longrightarrow \mathrm{Cl}_{2}(\mathbb{K})$, where $\mathbb{K}$ is an unramified extension of $\mathbb{k}$ within $\mathbb{k}_{2}^{(1)}$. The main results of this paper are Theorems 2 and 3 below; whereas Theorem 1 is proved in [2], [5] and [17].

2.1. Unramified extensions of $k$. The first and the second assertions of the following theorem hold according to [17] and [5] respectively, the others are shown in [2].

Theorem 1. Let $p_{1}, p_{2}$ be as above.

(1) The 2 -class groups of $k_{0}, \bar{k}_{0}$ are of type $(2,2)$.

(2) The 2-class group, $\mathrm{Cl}_{2}(\mathrm{k})$, of $\mathbb{k}$ is of type $(2,2,2)$.

(3) The discriminant of $\mathbb{k}$ is: $\operatorname{disc}(\mathbb{k})=\operatorname{disc}(k) \cdot \operatorname{disc}\left(k_{0}\right) \cdot \operatorname{disc}\left(\bar{k}_{0}\right)=2^{8} p_{1}^{2} p_{2}^{2}$.

(4) $\mathrm{k}$ has seven unramified quadratic extensions within its Hilbert 2-class field $\mathrm{k}_{2}^{(1)}$. They are given by:

$$
\begin{gathered}
\mathbb{K}_{1}=\mathbb{k}\left(\sqrt{p_{1}}\right), \quad \mathbb{K}_{2}=\mathbb{k}_{k}\left(\sqrt{p_{2}}\right), \quad \mathbb{K}_{3}=\mathbb{k}_{k}(\sqrt{2}), \\
\mathbb{K}_{4}=\mathbb{k}\left(\sqrt{\pi_{1} \pi_{3}}\right), \quad \mathbb{K}_{5}=\mathbb{k}\left(\sqrt{\pi_{1} \pi_{4}}\right), \mathbb{K}_{6}=\mathbb{k}\left(\sqrt{\pi_{2} \pi_{3}}\right) \text { and } \mathbb{K}_{7}=\mathbb{k}\left(\sqrt{\pi_{2} \pi_{4}}\right) .
\end{gathered}
$$

(5) $\mathbb{K}_{1}, \mathbb{K}_{2}, \mathbb{K}_{3}$ are intermediate fields between $\mathbb{k}$ and its genus field $\mathbb{k}^{(*)}$. The fields $\mathbb{K}_{4} \simeq \mathbb{K}_{7}$ and $\mathbb{K}_{5} \simeq \mathbb{K}_{6}$ are pairwise conjugate and thus isomorphic. Consequently $\mathbb{K}_{1}, \mathbb{K}_{2}, \mathbb{K}_{3}$ are absolutely abelian, whereas $\mathbb{K}_{4}, \mathbb{K}_{5}, \mathbb{K}_{6}, \mathbb{K}_{7}$ are non-normal over $\mathbb{Q}$.

(6) $\mathrm{k}$ has seven unramified bicyclic biquadratic extensions within its Hilbert 2 class field $\mathbb{k}_{2}^{(1)}$. One of them is

$$
\mathbb{L}_{1}=\mathbb{K}_{1} \cdot \mathbb{K}_{2} \cdot \mathbb{K}_{3}=\mathbb{k}^{(*)}=\mathbb{Q}\left(\sqrt{p_{1}}, \sqrt{p_{2}}, \sqrt{q}, \sqrt{-1}\right),
$$

the absolute genus field of $\mathbb{k}$ and the others are given by:

$$
\mathbb{L}_{2}=\mathbb{K}_{1} \cdot \mathbb{K}_{4} \cdot \mathbb{K}_{6}, \quad \mathbb{L}_{3}=\mathbb{K}_{1} \cdot \mathbb{K}_{5} \cdot \mathbb{K}_{7}, \quad \mathbb{L}_{4}=\mathbb{K}_{2} \cdot \mathbb{K}_{4} \cdot \mathbb{K}_{5} \text { and } \mathbb{L}_{5}=\mathbb{K}_{2} \cdot \mathbb{K}_{6} \cdot \mathbb{K}_{7}
$$

are non-normal over $\mathbb{Q} ;$ moreover $\mathbb{L}_{2} \simeq \mathbb{L}_{3}$ and $\mathbb{L}_{4} \simeq \mathbb{L}_{5}$.

$$
\mathbb{L}_{6}=\mathbb{K}_{3} \cdot \mathbb{K}_{4} \cdot \mathbb{K}_{7} \text { and } \mathbb{L}_{7}=\mathbb{K}_{3} \cdot \mathbb{K}_{5} \cdot \mathbb{K}_{6} \text { are absolutely Galois. }
$$


2.2. Structure of $G=\operatorname{Gal}\left(\mathbb{k}_{2}^{(2)} / \mathbb{k}\right)$. Let $\mathcal{H}_{0}$ (resp. $\mathcal{H}_{1}, \mathcal{H}_{2}$ ) denote the prime ideal of $\mathbb{k}$ lying above $1+i$ (resp. $\left.\pi_{1}, \pi_{2}\right)$. Write $q=q\left(\mathbb{K}_{3}^{+} / \mathbb{Q}\right)$ for simplicity.

Theorem 2. Keep the preceding assumptions. Then

(1) $\mathrm{Cl}_{2}(\mathbb{k})=\left\langle\left[\mathcal{H}_{0}\right],\left[\mathcal{H}_{1}\right],\left[\mathcal{H}_{2}\right]\right\rangle \simeq(2,2,2)$.

(2) $\mathrm{Cl}_{2}\left(\mathbb{K}_{3}\right)$ is of type $\left\{\begin{array}{l}\left(2^{m}, 2^{n+1}\right) \text { if } q=1, \\ \left(2^{\min (m, n+1)}, 2^{\max (m+1, n+2)}\right) \text { if } q=2,\end{array}\right.$

where $n$ and $m$ are determined by $2^{m+1}=h\left(-p_{1} p_{2}\right), m \geq 2$, and $2^{n}=$ $h\left(p_{1} p_{2}\right), n \geq 1$; and either $n \geq 3$ or $m \geq 3$.

(3) The length of the 2-class field tower of $\mathrm{k}$ is 2 .

(4) $G=\operatorname{Gal}\left(\mathbb{k}_{2}^{(2)} / \mathbb{k}\right)$ is given by:

(i) If $q=1$, then

$$
\begin{gathered}
G=\left\langle\rho, \tau, \sigma: \rho^{4}=\sigma^{2^{m}}=\tau^{2^{n+1}}=1, \rho^{2}=\psi,[\tau, \sigma]=1,\right. \\
\left.[\rho, \sigma]=\sigma^{2},[\rho, \tau]=\tau^{2}\right\rangle \text {, where } \\
\psi=\left\{\begin{array}{l}
\sigma^{2^{m-1}} \text { if }\left(\frac{p_{1}}{p_{2}}\right)=1 \text { and } N\left(\varepsilon_{p_{1} p_{2}}\right)=1, \\
\tau^{2^{n}} \sigma^{2^{m-1}} \text { if }\left(\frac{p_{1}}{p_{2}}\right)=-1 \text { or }\left(\frac{p_{1}}{p_{2}}\right)=1 \text { and } N\left(\varepsilon_{p_{1} p_{2}}\right)=-1 .
\end{array}\right.
\end{gathered}
$$

(ii) If $q=2$, then

$$
\begin{aligned}
G=\left\langle\rho, \tau, \sigma: \rho^{4}=\sigma^{2^{m+1}}=\tau^{2^{n+2}}=1, \sigma^{2^{m}}=\tau^{2^{n+1}}\right. \\
\left.\rho^{2}=\tau^{2^{n}} \sigma^{2^{m-1}},[\tau, \sigma]=1,[\rho, \sigma]=\sigma^{-2},[\rho, \tau]=\tau^{2}\right\rangle .
\end{aligned}
$$

(5) The derived group of $G$ is $G^{\prime}=\left\langle\sigma^{2}, \tau^{2}\right\rangle$ and $\mathbf{C} l_{2}\left(\mathrm{k}_{2}^{(1)}\right)$ is of type

$$
\left\{\begin{array}{l}
\left(2^{m-1}, 2^{n}\right) \text { if } q=1, \\
\left(2^{\min (m, n+1)-1}, 2^{\max (m+1, n+2)-1}\right)=\left(2,2^{n+1}\right) \text { if } q=2 .
\end{array}\right.
$$

(6) The coclass of $G$ is 3 and its nilpotency class is

$$
\left\{\begin{array}{l}
\max (n, m-1)+1 \text { if } q=1, \\
\max (n+1, m)+1 \text { if } q=2 .
\end{array}\right.
$$

2.3. Abelian type invariants and capitulation kernels. Let $N_{j}$ denote the subgroup $N_{\mathbb{K}_{j} / \mathbb{k}}\left(\mathbf{C} l_{2}\left(\mathbb{K}_{j}\right)\right)$ of $\mathbf{C} l_{2}(\mathbb{k})$ and $\kappa_{\mathbb{K}}$ denote the kernel of the natural class extension homomorphism $j_{\mathbb{K} / \mathbb{k}}: \mathbf{C} l_{2}(\mathbb{k}) \longrightarrow \mathbf{C} l_{2}(\mathbb{K})$, where $\mathbb{K}$ is an unramified extension of $\mathbb{k}_{\mathrm{k}}$ within $\mathrm{k}_{2}^{(1)}$.

Theorem 3. Let $2^{n}=h\left(p_{1} p_{2}\right), 2^{m+1}=h\left(-p_{1} p_{2}\right)$, where $n \geq 1$ and $m \geq 2$.

(1) $\# \kappa_{\mathbb{K}_{j}}=4$, for all $j \neq 3$. If $j=3$, then $\# \kappa_{\mathbb{K}_{3}}=\left\{\begin{array}{l}4 \text { if } q=1, \\ 2 \text { if } q=2 .\end{array}\right.$ 
(2) All the extensions $\mathbb{K}_{j}$ satisfy Taussky's condition $(A)$ i.e. $\# \kappa_{\mathbb{K}_{j}} \cap N_{j}>1$, for details see [16].

(3) The order of $\kappa_{\mathbb{L}_{j}}$ is 8 (total 2-capitulation), for all $j$, and $\mathbb{L}_{j}$ are of type $(A)$.

(4) The abelian type invariants of the 2 -class groups $\mathbf{C} l_{2}\left(\mathbb{K}_{j}\right)$ are given by:

(i) $\mathbf{C} l_{2}\left(\mathbb{K}_{1}\right) \simeq \mathbf{C} l_{2}\left(\mathbb{K}_{2}\right) \simeq\left\{\begin{array}{l}(2,2,2) \text { if }\left(\frac{p_{1}}{p_{2}}\right)=1, \\ (2,4) \text { otherwise. }\end{array}\right.$

(ii) If $\left(\frac{p_{1}}{p_{2}}\right)=1$, then $\mathbf{C} l_{2}\left(\mathbb{K}_{4}\right), \mathbf{C} l_{2}\left(\mathbb{K}_{5}\right), \mathbf{C l} l_{2}\left(\mathbb{K}_{6}\right)$ and $\mathbf{C} l_{2}\left(\mathbb{K}_{7}\right)$ are of type $(2,2,2)$ if $\left(\frac{\pi_{1}}{\pi_{3}}\right)=-1$, and of type $(2,4)$ otherwise.

(iii) Assume $\left(\frac{p_{1}}{p_{2}}\right)=-1$.

$$
\begin{aligned}
& \text { If }\left(\frac{\pi_{1}}{\pi_{3}}\right)=-1 \text {, then }\left\{\begin{array}{l}
\mathbf{C} l_{2}\left(\mathbb{K}_{4}\right) \simeq \mathbf{C} l_{2}\left(\mathbb{K}_{7}\right) \simeq(2,4), \\
\mathbf{C} l_{2}\left(\mathbb{K}_{5}\right) \simeq \mathbf{C} l_{2}\left(\mathbb{K}_{6}\right) \simeq(2,2,2) .
\end{array}\right. \\
& \text { If }\left(\frac{\pi_{1}}{\pi_{3}}\right)=1 \text {, then }\left\{\begin{array}{l}
\mathbf{C} l_{2}\left(\mathbb{K}_{4}\right) \simeq \mathbf{C} l_{2}\left(\mathbb{K}_{7}\right) \simeq(2,2,2), \\
\mathbf{C} l_{2}\left(\mathbb{K}_{5}\right) \simeq \mathbf{C} l_{2}\left(\mathbb{K}_{6}\right) \simeq(2,4) .
\end{array}\right.
\end{aligned}
$$

(5) The abelian type invariants of the 2-class groups $\mathbf{C} l_{2}\left(\mathbb{L}_{j}\right)$ are given by:

(i) $\mathbf{C} l_{2}\left(\mathbb{L}_{1}\right)=\mathbf{C} l_{2}\left(\mathbb{k}^{(*)}\right) \simeq\left\{\begin{array}{l}\left(2^{m}, 2^{n}\right) \text { if } q=1, \\ \left(2^{\min (m, n)}, 2^{\max (m+1, n+1)}\right) \text { if } q=2 .\end{array}\right.$

(ii) If $\left(\frac{p_{1}}{p_{2}}\right)=-1$ or $\left(\frac{p_{1}}{p_{2}}\right)=\left(\frac{\pi_{1}}{\pi_{3}}\right)=1$, then $\mathbf{C} l_{2}\left(\mathbb{L}_{2}\right), \mathbf{C} l_{2}\left(\mathbb{L}_{3}\right), \mathbf{C} l_{2}\left(\mathbb{L}_{4}\right)$ and $\mathbf{C} l_{2}\left(\mathbb{L}_{5}\right)$ are of type $(2,4)$.

If $\left(\frac{p_{1}}{p_{2}}\right)=-\left(\frac{\pi_{1}}{\pi_{3}}\right)=1$, then $\mathbf{C} l_{2}\left(\mathbb{L}_{2}\right), \mathbf{C} l_{2}\left(\mathbb{L}_{3}\right), \mathbf{C} l_{2}\left(\mathbb{L}_{4}\right)$ and $\mathbf{C} l_{2}\left(\mathbb{L}_{5}\right)$ are of type $(2,2,2)$.

(iii) (a) Assume $q=2$, so $\mathbf{C} l_{2}\left(\mathbb{L}_{6}\right)$ and $\mathbf{C} l_{2}\left(\mathbb{L}_{7}\right)$ are of type $\left(2,2^{n+2}\right)$ if $\left(\frac{p_{1}}{p_{2}}\right)=1$, otherwise we have:

$\mathbf{C} l_{2}\left(\mathbb{L}_{6}\right) \simeq\left\{\begin{array}{l}(4,4) \text { if }\left(\frac{\pi_{1}}{\pi_{3}}\right)=1, \\ (2,8) \text { if }\left(\frac{\pi_{1}}{\pi_{3}}\right)=-1,\end{array} \quad \mathbf{C} l_{2}\left(\mathbb{L}_{7}\right) \simeq\left\{\begin{array}{l}(2,8) \text { if }\left(\frac{\pi_{1}}{\pi_{3}}\right)=1, \\ (4,4) \text { if }\left(\frac{\pi_{1}}{\pi_{3}}\right)=-1 .\end{array}\right.\right.$

(b) Assume $q=1$.

$$
\begin{aligned}
& \text { If }\left(\frac{1+i}{\pi_{1}}\right)\left(\frac{1+i}{\pi_{3}}\right)=1, \text { then }\left\{\begin{array}{l}
\mathbf{C} l_{2}\left(\mathbb{L}_{6}\right) \simeq\left(2^{m-1}, 2^{n+1}\right), \\
\mathbf{C} l_{2}\left(\mathbb{L}_{7}\right) \simeq\left(2^{\min (m-1, n)}, 2^{\max (m, n+1)}\right) .
\end{array}\right. \\
& \text { If }\left(\frac{1+i}{\pi_{1}}\right)\left(\frac{1+i}{\pi_{3}}\right)=-1, \text { then }\left\{\begin{array}{l}
\mathbf{C} l_{2}\left(\mathbb{L}_{6}\right) \simeq\left(2^{\min (m-1, n)}, 2^{\max (m, n+1)}\right), \\
\mathbf{C} l_{2}\left(\mathbb{L}_{7}\right) \simeq\left(2^{m-1}, 2^{n+1}\right) .
\end{array}\right.
\end{aligned}
$$

\section{Preliminary Results}

Let $p_{1}, p_{2}$ be different primes satisfying the conditions mentioned at the beginning of $\S 2$, and set $k_{1}=\mathbb{Q}\left(\sqrt{p_{1} p_{2}}\right), \bar{k}_{1}=\mathbb{Q}\left(\sqrt{-p_{1} p_{2}}\right)$. Put $\varepsilon_{2 p_{1} p_{2}}=x+y \sqrt{2 p_{1} p_{2}}$ 
and $\varepsilon_{p_{1} p_{2}}=a+b \sqrt{p_{1} p_{2}}$. Let $\left(\frac{g, h}{p}\right)$ denote the quadratic Hilbert symbol for the prime $p$.

Lemma 1. Let $\varepsilon_{d}$ denote the fundamental unit of $k_{0}$. Then

(i) $N\left(\varepsilon_{d}\right)=-1$.

(ii) If $N\left(\varepsilon_{p_{1} p_{2}}\right)=1$, then $2 p_{1}(a \pm 1)$ (i.e. $2 p_{2}(a \mp 1)$ ) is a square in $\mathbb{N}$.

Proof. (i) See [5].

(ii) As $N\left(\varepsilon_{p_{1} p_{2}}\right)=1$, so $\left(\frac{p_{1}}{p_{2}}\right)=1$ and $a^{2}-1=b^{2} p_{1} p_{2}$, so:

(a) If $a \pm 1$ is a square in $\mathbb{N}$, then $\left(\frac{2}{p_{1}}\right)=-1$, which is false.

(b) If $p_{1}(a \pm 1)$ is a square in $\mathbb{N}$, then $\left(\frac{p_{1}}{p_{2}}\right)=\left(\frac{2}{p_{1}}\right)=-1$, which is absurd. And the result derived.

Lemma 2. If $\left(\frac{p_{1}}{p_{2}}\right)=1$, then $\left(\frac{p_{1}}{p_{2}}\right)_{4}\left(\frac{p_{2}}{p_{1}}\right)_{4}=\left(\frac{\pi_{1}}{\pi_{3}}\right)$.

Proof. From [17] we get $\left(\frac{p_{1}}{p_{2}}\right)_{4}\left(\frac{p_{2}}{p_{1}}\right)_{4}=\left(\frac{p_{1}}{a c+b d}\right)$, where $p_{1}=a^{2}+b^{2}$ and $p_{2}=$ $c^{2}+d^{2}$; on the other hand, according to [11] we have $\left(\frac{p_{1}}{a c+b d}\right)=\left(\frac{\pi_{1}}{\pi_{3}}\right)$, which implies the result.

Lemma 3. Put $\mathbb{k}_{\mathrm{k}}=\mathbb{Q}\left(\sqrt{2 p_{1} p_{2}}, i\right)$ and $\mathbb{K}_{3}=\mathbb{Q}\left(\sqrt{2}, \sqrt{p_{1} p_{2}}, i\right)$. Then

(1) $\left\{\varepsilon_{2 p_{1} p_{2}}\right\}$ is a FSU of $\mathrm{k}$.

(2) If $N\left(\varepsilon_{p_{1} p_{2}}\right)=1$ or $N\left(\varepsilon_{p_{1} p_{2}}\right)=-1$ and $\sqrt{\varepsilon_{2} \varepsilon_{p_{1} p_{2}} \varepsilon_{2 p_{1} p_{2}}} \notin \mathbb{K}_{3}^{+}$, then

(i) $\left\{\varepsilon_{2}, \varepsilon_{p_{1} p_{2}}, \varepsilon_{2 p_{1} p_{2}}\right\}$ is a FSU of both $\mathbb{K}_{3}^{+}$and $\mathbb{K}_{3}$.

(ii) $q=1, q\left(\mathbb{K}_{3} / \mathbb{Q}\right)=2$ and $h\left(\mathbb{K}_{3}\right)=h\left(p_{1} p_{2}\right) h\left(-p_{1} p_{2}\right)$.

(3) If $N\left(\varepsilon_{p_{1} p_{2}}\right)=-1$ and $\sqrt{\varepsilon_{2} \varepsilon_{p_{1} p_{2}} \varepsilon_{2 p_{1} p_{2}}} \in \mathbb{K}_{3}^{+}$, then

(i) $\left\{\varepsilon_{2}, \varepsilon_{p_{1} p_{2}}, \sqrt{\varepsilon_{2} \varepsilon_{p_{1} p_{2}} \varepsilon_{2 p_{1} p_{2}}}\right\}$ is a FSU of both $\mathbb{K}_{3}^{+}$and $\mathbb{K}_{3}$.

(ii) $q=2, q\left(\mathbb{K}_{3} / \mathbb{Q}\right)=4$ and $h\left(\mathbb{K}_{3}\right)=2 h\left(p_{1} p_{2}\right) h\left(-p_{1} p_{2}\right)$.

Proof. (1) As $N\left(\varepsilon_{2 p_{1} p_{2}}\right)=-1$, so if $N\left(\varepsilon_{p_{1} p_{2}}\right)=-1$ we get, according to [1, Applications 1), p.114], that $\left\{\varepsilon_{2 p_{1} p_{2}}\right\}$ is a FSU of $\mathbb{k}$.

(2) Assume that $N\left(\varepsilon_{p_{1} p_{2}}\right)=1$. As $N\left(\varepsilon_{2}\right)=N\left(\varepsilon_{2 p_{1} p_{2}}\right)=-1$, so $\varepsilon_{2}, \varepsilon_{2 p_{1} p_{2}}$, $\varepsilon_{2} \varepsilon_{p_{1} p_{2}}, \varepsilon_{2} \varepsilon_{2 p_{1} p_{2}}, \varepsilon_{p_{1} p_{2}} \varepsilon_{2 p_{1} p_{2}}$ and $\varepsilon_{2} \varepsilon_{p_{1} p_{2}} \varepsilon_{2 p_{1} p_{2}}$ are not squares in $\mathbb{K}_{3}^{+}$, else by taking a suitable norm we get $i \in \mathbb{K}_{3}^{+}$, which is false. Furthermore $(2+\sqrt{2}) \varepsilon_{2}^{i} \varepsilon_{p_{1} p_{2}}^{j} \varepsilon_{2 p_{1} p_{2}}^{k}$ can not be a square in $\mathbb{K}_{3}^{+}$, for all $\mathrm{i}, \mathrm{j}$ and $\mathrm{k}$ in $\{0,1\}$, as otherwise with some $\alpha \in \mathbb{K}_{3}^{+}$ we would have $\alpha^{2}=(2+\sqrt{2}) \varepsilon_{2}^{i} \varepsilon_{p_{1} p_{2}}^{j} \varepsilon_{2 p_{1} p_{2}}^{k}$, so $\left(N_{\mathbb{K}+/ \mathbb{Q}\left(\sqrt{p_{1} p_{2}}\right)}(\alpha)\right)^{2}=2(-1)^{i+k} \varepsilon_{p_{1} p_{2}}^{2 j}$, yielding that $\sqrt{2} \in \mathbb{Q}\left(\sqrt{p_{1} p_{2}}\right)$, which is absurd. 
Put $\varepsilon_{p_{1} p_{2}}=a+b \sqrt{p_{1} p_{2}}$; as $2 p_{1}(a \pm 1)$ is a square in $\mathbb{N}$, thus $\sqrt{2 \varepsilon_{p_{1} p_{2}}}=b_{1} \sqrt{2 p_{1}}+$ $b_{2} \sqrt{2 p_{2}}$, where $b_{i} \in \mathbb{Z}$; so $\varepsilon_{p_{1} p_{2}}$ is not a square in $\mathbb{K}_{3}^{+}$; hence $\left\{\varepsilon_{2}, \varepsilon_{p_{1} p_{2}}, \varepsilon_{2 p_{1} p_{2}}\right\}$ is a FSU of $\mathbb{K}_{3}^{+}$, which implies that $q=1$. Thus from [1, Proposition 3, p.112] we get $\left\{\varepsilon_{2}, \varepsilon_{p_{1} p_{2}}, \varepsilon_{p_{1} p_{2} q}\right\}$ is a FSU of $\mathbb{K}_{3}$, we infer that $q\left(\mathbb{K}_{3} / \mathbb{Q}\right)=2$, since $\sqrt{i} \in \mathbb{K}_{3}$.

If $N\left(\varepsilon_{p_{1} p_{2}}\right)=-1$, then the results are guaranteed by [1, Propositions 8, 15]. In the end, under our conditions, P.Kaplan states in [17] that $h\left(2 p_{1} p_{2}\right)=h\left(-2 p_{1} p_{2}\right)=4$, therefore the class number formula yields that $h\left(\mathbb{K}_{3}^{+}\right)=h\left(p_{1} p_{2}\right)$ and $h\left(\mathbb{K}_{3}\right)=$ $h\left(p_{1} p_{2}\right) h\left(-p_{1} p_{2}\right)$.

(3) If $N\left(\varepsilon_{p_{1} p_{2}}\right)=-1$ and $\sqrt{\varepsilon_{2} \varepsilon_{p_{1} p_{2}} \varepsilon_{2 p_{1} p_{2}}} \in \mathbb{K}_{3}^{+}$, then the results are also deduced from [1, Propositions 8, 15] and the class number formula implies (3)(iii).

Lemma 4. Let $\kappa_{\mathbb{K}_{3}}$ denote the set of classes of $\mathbf{C l} l_{2}(\mathbb{k})$ that capitulate in $\mathbb{K}_{3}$, then $\kappa_{\mathbb{K}_{3}}=\left\{\begin{array}{l}\left\langle\left[\mathcal{H}_{0}\right]\right\rangle \text { if } q=2, \\ \left\langle\left[\mathcal{H}_{0}\right],\left[\mathcal{H}_{1} \mathcal{H}_{2}\right]\right\rangle \text { if } q=1\end{array}\right.$

Proof. From Lemma 3 we get $E_{\mathbb{k}}=\left\langle i, \varepsilon_{2 p_{1} p_{2}}\right\rangle$ and $E_{\mathbb{K}_{3}}=\left\langle\sqrt{i}, \varepsilon_{2}, \varepsilon_{p_{1} p_{2}}, \varepsilon_{2 p_{1} p_{2}}\right\rangle$ or $E_{\mathbb{K}_{3}}=\left\langle\sqrt{i}, \varepsilon_{2}, \varepsilon_{p_{1} p_{2}}, \sqrt{\varepsilon_{2} \varepsilon_{p_{1} p_{2}} \varepsilon_{p_{1} p_{2} q}}\right\rangle$, according as $q=1$ or 2 . Therefore $N_{\mathbb{K}_{3} / \mathbb{k}}\left(E_{\mathbb{K}_{3}}\right)=$ $\left\langle i, \varepsilon_{2 p_{1} p_{2}}^{2}\right\rangle$ or $\left\langle i, \varepsilon_{2 p_{1} p_{2}}\right\rangle$, thus

$$
\left[E_{\mathbb{k}}: N_{\mathbb{K}_{3} / \mathbb{k}}\left(E_{\mathbb{K}_{3}}\right)\right]=\left\{\begin{array}{l}
2, \text { if } q=1, \\
1, \text { if } q=2 ;
\end{array} \text { hence } \# \kappa_{\mathbb{K}_{3}}=\left\{\begin{array}{l}
4, \text { if } q=1 \\
2, \text { if } q=2
\end{array}\right.\right.
$$

Moreover, it is easy to see that $\sqrt{(1+i) \varepsilon_{2}}=\frac{1}{2}(2+(1+i) \sqrt{2})$, so there exists $\beta \in \mathbb{K}_{3}$ such that $\mathcal{H}_{0}^{2}=(1+i)=\left(\beta^{2}\right)$, this implies that $\mathcal{H}_{0}$ capitulates in $\mathbb{K}_{3}$. Consequently, if $q=2$, then $\kappa_{\mathbb{K}_{3}}=\left\langle\left[\mathcal{H}_{0}\right]\right\rangle$.

Suppose $q=1$, then we have two cases to discuss:

(a) If $N\left(\varepsilon_{p_{1} p_{2}}\right)=1$, then by Lemma 1, we get $\sqrt{2 \varepsilon_{p_{1} p_{2}}}=b_{1} \sqrt{2 p_{1}}+b_{2} \sqrt{2 p_{2}}$, where $b=2 b_{1} b_{2}$, from which we deduce that $2 p_{1} \varepsilon_{p_{1} p_{2}}$ is a square in $\mathbb{K}_{3}$, thus there exists $\alpha \in \mathbb{K}_{3}$ such that $\left(2 p_{1}\right)=\left(\alpha^{2}\right)$. On the other hand, $\left(\mathcal{H}_{1} \mathcal{H}_{2}\right)^{2}=\left(p_{1}\right)$ and $(2)=(2 i)=(1+i)^{2}$, hence $\mathcal{H}_{1} \mathcal{H}_{2}=\left(\frac{\alpha}{1+i}\right)$, which implies that $\mathcal{H}_{1} \mathcal{H}_{2}$ capitulates in $\mathbb{K}_{3}$.

(b) If $N\left(\varepsilon_{p_{1} p_{2}}\right)=-1$, then there exist an even integer $a$ and an odd integer $b$ such that $\varepsilon_{p_{1} p_{2}}=a+b \sqrt{p_{1} p_{2}}$, so $a^{2}+1=b^{2} p_{1} p_{2}$, since $p_{1} p_{2} \equiv 1(\bmod 8)$. Therefore:

$$
\left\{\begin{array} { l } 
{ a \mp i = i b _ { 1 } ^ { 2 } \pi _ { 1 } \pi _ { 3 } , } \\
{ a \pm i = - i b _ { 2 } ^ { 2 } \pi _ { 2 } \pi _ { 4 } , }
\end{array} \text { or } \left\{\begin{array}{l}
a \mp i=i b_{1}^{2} \pi_{1} \pi_{4} \\
a \pm i=-i b_{2}^{2} \pi_{2} \pi_{3}
\end{array}\right.\right.
$$




$$
\text { hence } \left.\begin{array}{l}
\sqrt{\varepsilon_{p_{1} p_{2}}}=z_{1} \sqrt{\pi_{1} \pi_{3}}+z_{2} \sqrt{\pi_{2} \pi_{4}} \text { or } \\
\sqrt{\varepsilon_{p_{1} p_{2}}}=z_{1} \sqrt{\pi_{1} \pi_{4}}+z_{2} \sqrt{\pi_{2} \pi_{3}},
\end{array}\right\}
$$

where $z_{2}$ is the conjugate of $z_{1}$ in $\frac{1}{2} \mathbb{Z}[i]$.

Similarly, as $N\left(\varepsilon_{2 p_{1} p_{2}}\right)=-1$, so there exist $x, y$ in $\mathbb{N}$ such that $x^{2}+1=2 p_{1} p_{2} y^{2}$, and

$$
\left.\begin{array}{rl}
\sqrt{\varepsilon_{2 p_{1} p_{2}}} & \left.=y_{1} \sqrt{(1+i) \pi_{1} \pi_{3}}+y_{2} \sqrt{(1-i) \pi_{2} \pi_{4}}\right), \text { or } \\
\left.\sqrt{\varepsilon_{2 p_{1} p_{2}}}=y_{1} \sqrt{(1+i) \pi_{1} \pi_{4}}+y_{2} \sqrt{(1-i) \pi_{2} \pi_{3}}\right), \text { or } \\
\left.\sqrt{2 \varepsilon_{2 p_{1} p_{2}}}=y_{1} \sqrt{(1+i) \pi_{1} \pi_{3}}+y_{2} \sqrt{(1-i) \pi_{2} \pi_{4}}\right), \text { or } \\
\left.\sqrt{2 \varepsilon_{2 p_{1} p_{2}}}=y_{1} \sqrt{(1+i) \pi_{1} \pi_{3}}+y_{2} \sqrt{(1-i) \pi_{2} \pi_{4}}\right),
\end{array}\right\}
$$

where $y_{i}$ are in $\mathbb{Z}[i]$ or $\frac{1}{2} \mathbb{Z}[i]$.

Finally, Note that:

$$
\sqrt{2 \varepsilon_{1}}=\sqrt{1+i}+\sqrt{1-i}
$$

So by multiplying the equalities (11), (2) and (3) we get

$$
\begin{aligned}
& \sqrt{\varepsilon_{1} \varepsilon_{2} \varepsilon_{3}}=\alpha+\beta \sqrt{2}+\gamma \sqrt{p_{1} p_{2}}+\delta \sqrt{2 p_{1} p_{2}} \in \mathbb{Q}\left(\sqrt{2}, \sqrt{p_{1} p_{2}}\right) \text { or } \\
& \sqrt{\varepsilon_{1} \varepsilon_{2} \varepsilon_{3}}=\alpha \sqrt{p_{1}}+\beta \sqrt{p_{2}}+\gamma \sqrt{2 p_{1}}+\delta \sqrt{2 p_{2}} \notin \mathbb{Q}\left(\sqrt{2}, \sqrt{p_{1} p_{2}}\right),
\end{aligned}
$$

where $\alpha, \beta, \gamma$ and $\delta$ are in $\mathbb{Q}$.

As $q=1$, so $\varepsilon_{2} \varepsilon_{p_{1} p_{2}} \varepsilon_{p_{1} p_{2} q}$ is not a square in $\mathbb{K}_{3}^{+}$, hence $p_{1} \varepsilon_{2} \varepsilon_{p_{1} p_{2}} \varepsilon_{p_{1} p_{2} q}$ is a square in $\mathbb{K}_{3}$; which yields that $\mathcal{H}_{1} \mathcal{H}_{2}$ capitulates in $\mathbb{K}_{3}$. Thus $\kappa_{\mathbb{K}_{3}}=\left\langle\left[\mathcal{H}_{0}\right],\left[\mathcal{H}_{1} \mathcal{H}_{2}\right]\right\rangle$.

Proposition 1 ([4]). Let $p_{1} \equiv p_{2} \equiv 1(\bmod 4)$ be different primes such that $\left(\frac{2}{p_{1}}\right)=\left(\frac{2}{p_{2}}\right)=\left(\frac{p_{1}}{p_{2}}\right)$. Then

$$
\left(\frac{p_{1} p_{2}}{2}\right)_{4}\left(\frac{2 p_{1}}{p_{2}}\right)_{4}\left(\frac{2 p_{2}}{p_{1}}\right)_{4}=\left(\frac{\pi_{1}}{\pi_{3}}\right)\left(\frac{1+i}{\pi_{1}}\right)\left(\frac{1+i}{\pi_{3}}\right) .
$$

Proposition $2\left([4)\right.$. Let $p_{1} \equiv p_{2} \equiv 1(\bmod 4)$ be different primes such that $\left(\frac{2}{p_{1}}\right)=\left(\frac{2}{p_{2}}\right)=\left(\frac{p_{1}}{p_{2}}\right)=-1$. Then the following assertions are equivalent:

(1) $\varepsilon_{2} \varepsilon_{p_{1} p_{2}} \varepsilon_{2 p_{1} p_{2}}$ is a square in $\mathbb{K}_{3}^{+}$.

(2) $\left(\frac{p_{1} p_{2}}{2}\right)_{4}\left(\frac{2 p_{1}}{p_{2}}\right)_{4}\left(\frac{2 p_{2}}{p_{1}}\right)_{4}=-1$.

(3) $q\left(\mathbb{K}_{3}^{+} / \mathbb{Q}\right)=2$.

The following results are deduced from $[9]$.

Theorem 4. Let $p_{1} \equiv p_{2} \equiv 5(\bmod 8)$ be different primes and put $F_{1}=\mathbb{Q}\left(\sqrt{p_{1} p_{2}}, i\right)$. 
(1) $\mathrm{Cl}_{2}\left(\bar{k}_{1}\right)$ is of type $\left(2,2^{m}\right), m \geq 2$. It is generated by $2=\left(2,1+\sqrt{-p_{1} p_{2}}\right)$, the prime ideal of $\bar{k}_{1}$ above 2 , and an ideal I of $\bar{k}_{1}$ of order $2^{m}$. Moreover

$$
\left\{\begin{array}{l}
I^{2^{m-1}} \sim \mathfrak{p}_{1} \text { if }\left(\frac{p_{1}}{p_{2}}\right)=1, \\
I^{2^{m-1}} \sim 2 \mathfrak{p}_{1} \text { if }\left(\frac{p_{1}}{p_{2}}\right)=-1 ;
\end{array}\right.
$$

where $\mathfrak{p}_{1}=\left(p_{1}, \sqrt{-p_{1} p_{2}}\right)$ is the prime ideal of $\bar{k}_{1}$ above $p_{1}$.

(2) $\mathrm{Cl}_{2}\left(k_{1}\right)$ is of type $\left(2^{n}\right), n \geq 1$, and it is generated by $2_{1}$, a prime ideal of $k_{1}$ above 2 .

(3) $\mathbf{C l}_{2}\left(F_{1}\right)$ is of 2-rank equal to 2 . It is generated by $I$ and $2_{F_{1}}$, where $2_{F_{1}}$ is a prime ideal of $F_{1}$ above 2 .

(4) If $\left(\frac{p_{1}}{p_{2}}\right)=-1$, then $\mathbf{C} l_{2}\left(F_{1}\right) \simeq\left(2^{n}, 2^{m}\right)$; and, in $\mathbf{C} l_{2}\left(F_{1}\right), I^{2^{m-1}} \sim 2_{F_{1}}^{2^{n}} \sim$ $\mathfrak{p}_{1} \nsim 1$.

(5) If $\left(\frac{p_{1}}{p_{2}}\right)=1$ and $N\left(\varepsilon_{p_{1} p_{2}}\right)=-1$, then

$$
\mathbf{C} l_{2}\left(F_{1}\right) \simeq\left(2^{\min (n, m-1)}, 2^{\max (m-1, n+1)}\right)
$$

and $I^{2^{m-1}} \sim 2_{F_{1}}^{2^{n}} \sim \mathfrak{p}_{1} \nsim 1$.

(6) If $\left(\frac{p_{1}}{p_{2}}\right)=1$ and $N\left(\varepsilon_{p_{1} p_{2}}\right)=1$, then $\mathbf{C} l_{2}\left(F_{1}\right) \simeq\left(2^{n+1}, 2^{m-1}\right)$; moreover $I^{2^{m-1}} \sim 2_{F_{1}}^{2^{n+1}} \sim \mathfrak{p}_{1} \sim 1$

Using the above theorem, we prove the following lemma.

Lemma 5. Let $\mathfrak{p}_{1} \mathcal{O}_{F_{1}}=\mathcal{P}_{1} \mathcal{P}_{2}$ and $p_{2} \mathcal{O}_{F_{1}}=\mathcal{P}_{3}^{2} \mathcal{P}_{4}^{2}$, then in $\mathbf{C} l_{2}\left(F_{1}\right)$ we have:

(i) If $\left(\frac{p_{1}}{p_{2}}\right)=-1$ or $\left(\frac{p_{1}}{p_{2}}\right)=1$ and $N\left(\varepsilon_{p_{1} p_{2}}\right)=-1$, then $\mathcal{P}_{1} \sim 2_{F_{1}}^{2^{n-1}} I^{2^{m-2}}$.

(ii) If $\left(\frac{p_{1}}{p_{2}}\right)=1$ and $N\left(\varepsilon_{p_{1} p_{2}}\right)=1$, then $\mathcal{P}_{1} \sim 2_{F_{1}}^{2^{n}} I^{2^{m-2}}$ or $\mathcal{P}_{1} \sim I^{2^{m-2}}$. Moreover $\mathcal{P}_{1} \mathcal{P}_{3} \sim 2_{F_{1}}^{2^{n}}$.

Proof. Let $p_{1} \mathcal{O}_{\mathbb{Q}(i)}=\pi_{1} \pi_{2}, p_{2} \mathcal{O}_{\mathbb{Q}(i)}=\pi_{3} \pi_{4}, \mathfrak{p}_{1} \mathcal{O}_{F_{1}}=\mathcal{P}_{1} \mathcal{P}_{2}$ and $\mathfrak{p}_{2} \mathcal{O}_{F_{1}}=\mathcal{P}_{3} \mathcal{P}_{4}$, where $\mathfrak{p}_{2}$ is the prime ideal of $\bar{k}_{1}$ above $p_{2}$, then $\left(\pi_{i}\right)=\mathcal{P}_{i}^{2}$, for all $i$. So, according to [3, Proposition 1], $\mathcal{P}_{i}$ are not principals in $F_{1}$ and they are of order two. On the other hand, as the 2-rank of $\mathbf{C} l_{2}\left(F_{1}\right)$ is 2 , thus $\mathcal{P}_{i} \in\left\langle\left[{ }_{2} F_{1}\right],[I]\right\rangle$.

(i) In this case, we have $\mathfrak{p}_{1} \nsim 1$, hence $\mathcal{P}_{1} \nsim \mathcal{P}_{2}$; note that the elements of order two in $\mathbf{C l}_{2}\left(F_{1}\right)$ are $2_{F_{1}}^{2^{n-1}} I^{2^{m-2}}, 2_{F_{1}}^{2^{n-1}} I^{-2^{m-2}}$ and $2_{F_{1}}^{2^{n}} \sim I^{2^{m-1}}$. Therefore $\mathcal{P}_{1}$ is equivalent to one of these three elements. As $\mathcal{P}_{1} \sim 2_{F_{1}}^{2^{n}} \sim I^{2^{m-1}}$ can not occur, if not we would have, by applying the norm $N_{F_{1} / \bar{k}_{1}}, \mathfrak{p}_{1} \sim I^{2^{m}} \sim 1$, which is false. Thus $\mathcal{P}_{1} \sim 2_{F_{1}}^{2^{n-1}} I^{2^{m-2}}$ and $\mathcal{P}_{2} \sim 2_{F_{1}}^{2^{n-1}} I^{-2^{m-2}}$ or $\mathcal{P}_{1} \sim 2_{F_{1}}^{2^{n-1}} I^{-2^{m-2}}$ and 
$\mathcal{P}_{2} \sim 2_{F_{1}}^{2^{n-1}} I^{2^{m-2}}$. Hence with out loss of generality we can choose $\mathcal{P}_{1} \sim 2_{F_{1}}^{2^{n-1}} I^{2^{m-2}}$.

(ii) In this case, we have $\mathfrak{p}_{1} \sim \mathfrak{p}_{2} \sim 1$, hence $\mathcal{P}_{1} \sim \mathcal{P}_{2}$ and $\mathcal{P}_{3} \sim \mathcal{P}_{4}$. On the other hand, according to [3, Proposition 1], $\mathcal{P}_{1} \mathcal{P}_{3}$ is not principal in $F_{1}$. To this end, note that the elements of order two in $\mathbf{C} l_{2}\left(F_{1}\right)$ are $2_{F_{1}}^{2^{n}} I^{2^{m-2}}, 2_{F_{1}}^{2^{n}}$ and $I^{-2^{m-2}}$. Therefore $\mathcal{P}_{1}$ is equivalent to one of these three elements. As $\mathcal{P}_{1} \sim 2_{F_{1}}^{2^{n}}$ can not occur, as otherwise, by applying the norm $N_{F_{1} / k_{1}}$, we get $\mathfrak{p}_{1} \sim 2^{2^{n}} \sim 1$, which is false. Thus $\mathcal{P}_{1} \sim I^{2^{m-2}}$ and $\mathcal{P}_{3} \sim 2_{F_{1}}^{2^{n}} I^{2^{m-2}}$ or $\mathcal{P}_{1} \sim 2_{F_{1}}^{2^{n-1}} I^{2^{m-2}}$ and $\mathcal{P}_{3} \sim I^{2^{m-2}}$. Hence $\mathcal{P}_{1} \mathcal{P}_{3} \sim 2_{F_{1}}^{2^{n}}$.

We conclude this section with the following lemma which gives the relationship between the unit index $q$ and the integers $n$ and $m$. It is a consequence of Lemma 3. Proposition 2 and the results in [6], [17].

Lemma 6. (1) Suppose $q=1$, so

(i) If $\left(\frac{p_{1}}{p_{2}}\right)=-1$, then $n=1$ and $m \geq 3$.

(ii) If $\left(\frac{p_{1}}{p_{2}}\right)=1$, then
(a) If $\left(\frac{p_{1}}{p_{2}}\right)_{4}\left(\frac{p_{2}}{p_{1}}\right)_{4}=-1$, then $n=1$ and $m \geq 3$.
(b) If $\left(\frac{p_{1}}{p_{2}}\right)_{4}\left(\frac{p_{2}}{p_{1}}\right)_{4}=1$, then $m=2$ and $n \geq 2$.

(2) Suppose $q=2$, so

(i) If $\left(\frac{p_{1}}{p_{2}}\right)=-1$, then $n=1$ and $m=2$.

(ii) If $\left(\frac{p_{1}}{p_{2}}\right)=1$, then $m=2$ and $n \geq 2$.

\section{Proofs of the main Results}

Recall first the following result from [12, p. 205].

Lemma 7. If $\mathcal{H}$ is an unramified ideal in some extension $\mathbb{K} / \mathbb{k}=\mathbb{k}(\sqrt{x}) / \mathbb{k}$, then the quadratic residue symbol is given by the Artin symbol $\varphi=\left(\frac{\mathbb{k}(\sqrt{x}) / \mathbb{k}}{\mathcal{H}}\right)$ as follows: $\left(\frac{x}{\mathcal{H}}\right)=\sqrt{x}^{\varphi-1}$.

4.1. Proof of Theorem 2, (1) The assertion $\mathbf{C l} l_{2}(\mathbb{k})=\left\langle\left[\mathcal{H}_{0}\right],\left[\mathcal{H}_{1}\right],\left[\mathcal{H}_{2}\right]\right\rangle \simeq(2,2,2)$ of Theorem 2 is proved in [5] and [3. In the following pages we will prove the other assertions.

(2) To prove the second assertion we will use the techniques that F. Lemmermeyer has used in some of his works see for example [9] or [10]. Consider the following diagram 


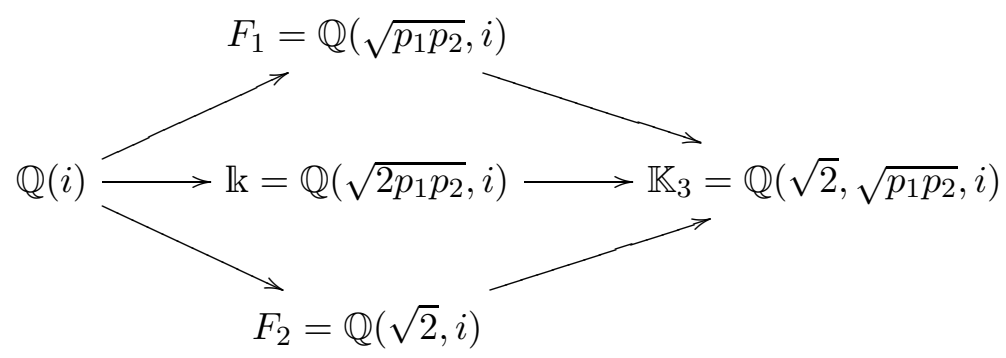

Figure 1. Subfields of $\mathbb{K}_{3} / \mathbb{Q}(i)$

Compute first $N_{\mathbb{K}_{3} / \mathbb{k}}\left(\mathbf{C l}_{2}\left(\mathbb{K}_{3}\right)\right)$. Recall that

$$
N_{\mathbb{K}_{3} / \mathbb{k}}\left(\mathbf{C l}_{2}\left(\mathbb{K}_{3}\right)\right)=\left\{[\mathcal{H}] \in \mathbf{C} l_{2}(\mathbb{k}) /\left(\frac{2}{[\mathcal{H}]}\right)=1\right\} .
$$

As $\mathcal{H}_{1}$ and $\mathcal{H}_{2}$ are unramified in $\mathbb{K}_{3} / \mathbb{k}=\mathbb{k}(\sqrt{2}) / \mathbb{k}=\mathbb{k}\left(\sqrt{p_{1} p_{2}}\right) / \mathbb{k}$, so Lemma 7 yields that $\left(\frac{2}{\mathcal{H}_{1} \mathcal{H}_{2}}\right)=\left(\frac{2}{\mathcal{H}_{1}}\right)\left(\frac{2}{\mathcal{H}_{2}}\right)=\left(\frac{2}{p_{1}}\right)\left(\frac{2}{p_{1}}\right)=1$. On the other hand, 2 ramifies completely in $\mathbb{k} / \mathbb{Q}$ and splits in $F_{1} / \mathbb{Q}$; moreover $\mathcal{H}_{0}$ is unramified in $\mathbb{K}_{3} / \mathbb{k}$, then $\mathcal{H}_{0}$ splits in $\mathbb{K}_{3}$ i.e. $\mathcal{H}_{0} \in N_{\mathbb{K}_{3} / \mathbb{k}}\left(\mathbf{C} l_{2}\left(\mathbb{K}_{3}\right)\right)$. Thus

$$
N_{\mathbb{K}_{3} / \mathbb{k}}\left(\mathbf{C l}_{2}\left(\mathbb{K}_{3}\right)\right)=\left\langle\left[\mathcal{H}_{0}\right],\left[\mathcal{H}_{1} \mathcal{H}_{2}\right]\right\rangle .
$$

To this end, it is easy to see that $\mathbb{K}_{3} / F_{1}$ and $\mathbb{K}_{3} / F_{2}$ are ramified extensions, whereas $\mathbb{K}_{3} / \mathbb{k}$ is not; so from the class field theory $\left[\mathbf{C} l_{2}(\mathbb{k}): N_{\mathbb{K}_{3} / \mathbb{k}}\left(\mathbf{C} l_{2}\left(\mathbb{K}_{3}\right)\right)\right]=2$, $\mathbf{C} l_{2}\left(F_{2}\right)=N_{\mathbb{K}_{3} / F_{2}}\left(\mathbf{C} l_{2}\left(\mathbb{K}_{3}\right)\right)$ and $\mathbf{C} l_{2}\left(F_{1}\right)=N_{\mathbb{K}_{3} / F_{1}}\left(\mathbf{C l}_{2}\left(\mathbb{K}_{3}\right)\right)$, hence Theorem 4 implies that

$$
N_{\mathbb{K}_{3} / F_{1}}\left(\mathbf{C l}_{2}\left(\mathbb{K}_{3}\right)\right)=\left\langle\left[2_{F_{1}}\right],[I]\right\rangle .
$$

Then there exists an ideal $\mathfrak{P} \in \mathbb{K}_{3}$ such that $N_{\mathbb{K}_{3} / F_{1}}(\mathfrak{P}) \sim I$ and $N_{\mathbb{K}_{3} / \mathbb{k}}(\mathfrak{P}) \in$ $\left\langle\left[\mathcal{H}_{0}\right],\left[\mathcal{H}_{1} \mathcal{H}_{2}\right]\right\rangle$. One shows that $N_{\mathbb{K}_{3} / \mathbb{k}}(\mathfrak{P}) \sim \mathcal{H}_{1} \mathcal{H}_{2}$ (see Lemma 8 below). We claim that

$$
\left\{\begin{array}{l}
\mathfrak{P}^{2} \sim I, \text { if } q=1, \\
\mathfrak{P}^{2} \sim \mathcal{H}_{1} \mathcal{H}_{2} I, \text { if } q=2 .
\end{array}\right.
$$

Let $t$ and $s$ be the elements of order 2 of $\operatorname{Gal}\left(\mathbb{K}_{3} / \mathbb{Q}(i)\right)$ which fix $F_{1}$ and $\mathbb{k}$, respectively. Using the identity $2+(1+t+s+t s)=(1+t)+(1+s)+(1+t s)$ of the group ring $\mathbb{Z}\left[\mathrm{Gal}\left(\mathbb{K}_{3} / \mathbb{Q}(i)\right)\right]$ and observing that the class numbers of $\mathbb{Q}(i), F_{2}$ are odd, we find that

$$
\mathfrak{P}^{2} \sim \mathfrak{P}^{1+t} \mathfrak{P}^{1+s} \mathfrak{P}^{1+t s} \sim \mathcal{H}_{1} \mathcal{H}_{2} I
$$


As $\mathcal{H}_{1} \mathcal{H}_{2} \sim 1$, in $\mathbf{C} l_{2}\left(\mathbb{K}_{3}\right)$, if $q=1$ (see Lemma 4), so the result claimed. Moreover $\mathfrak{p}_{1} \mathcal{O}_{\mathbb{k}}=\mathcal{H}_{1} \mathcal{H}_{2}$, so Lemma 4 yields that in $\mathbf{C} l_{2}\left(\mathbb{K}_{3}\right)$ we have

$$
\left\{\begin{array}{l}
\mathfrak{P}^{2^{m}} \sim I^{2^{m-1}} \sim \mathfrak{p}_{1} \sim 1, \text { if } q=1, \\
\mathfrak{P}^{2^{m}} \sim I^{2^{m-1}} \sim \mathfrak{p}_{1}, \text { if } q=2 .
\end{array}\right.
$$

On the other hand, ${ }_{2} F_{1}$ ramifies and $\mathcal{H}_{0}$ splits in $\mathbb{K}_{3}$, let $\mathfrak{A}$ be an ideal of $\mathbb{K}_{3}$ above ${ }^{2} F_{1}$, then $N_{\mathbb{K}_{3} / \mathbb{k}}(\mathfrak{A}) \sim \mathcal{H}_{0}$ and $N_{\mathbb{K}_{3} / F_{1}}(\mathfrak{A}) \sim{ }^{2} F_{1}$. Thus, in $\mathbf{C} l_{2}\left(\mathbb{K}_{3}\right)$, we have

$$
\mathfrak{A}^{2} \sim 2_{F_{1}} \text { and } \mathfrak{A}^{2^{n+1}} \sim 2_{F_{1}}^{2^{n}} .
$$

Recall that $\mathcal{H}_{j}$ and $\mathcal{P}_{j}$ coincide and remain inert in $\mathbb{K}_{3}$; moreover $\mathfrak{p}_{1} \mathcal{O}_{\mathbb{K}_{3}}=\mathcal{H}_{1} \mathcal{H}_{2} \mathcal{O}_{\mathbb{K}_{3}}=$ $\mathcal{P}_{1} \mathcal{P}_{2} \mathcal{O}_{\mathbb{K}_{3}}$ and $\mathfrak{p}_{2} \mathcal{O}_{\mathbb{K}_{3}}=\mathcal{H}_{3} \mathcal{H}_{4} \mathcal{O}_{\mathbb{K}_{3}}=\mathcal{P}_{3} \mathcal{P}_{4} \mathcal{O}_{\mathbb{K}_{3}}$, therefore:

- If $\left(\frac{p_{1}}{p_{2}}\right)=-1$ or $\left(\frac{p_{1}}{p_{2}}\right)=1$ and $N\left(\varepsilon_{p_{1} p_{2}}\right)=-1$, then Lemmas 3 , 4 and Theorem 4 imply that

$$
\left\{\begin{array}{l}
\mathfrak{A}^{2^{n+1}} \sim 2_{F_{1}}^{2^{n}} \sim 1, \text { if } q=1, \\
\mathfrak{A}^{2^{n+1}} \sim 2_{F_{1}}^{2^{n}} \nsim 1 \text { and } \mathfrak{A}^{2^{n+2}} \sim 2_{F_{1}}^{2^{n+1}} \sim 1, \text { if } q=2 .
\end{array}\right.
$$

- If $\left(\frac{p_{1}}{p_{2}}\right)=1$ and $N\left(\varepsilon_{p_{1} p_{2}}\right)=1$, then $q=1$, and Lemma 5 yields that $\mathcal{P}_{1} \mathcal{P}_{3} \sim 2_{F_{1}}^{2^{n}}$.

Let us prove that $\mathcal{P}_{1} \mathcal{P}_{3} \mathcal{O}_{\mathbb{K}_{3}}=\mathcal{H}_{1} \mathcal{H}_{3} \mathcal{O}_{\mathbb{K}_{3}}$ is principal. We know that $N\left(\varepsilon_{2 p_{1} p_{2}}\right)=$ -1 , so the decomposition uniqueness in $\mathbb{Z}[i]$ implies that there exist $y_{1}, y_{2}$ in $\mathbb{Z}[i]$ such that

$$
\begin{gathered}
\sqrt{\varepsilon_{d}}=\frac{1}{2}\left[y_{1}(1+i) \sqrt{(1 \pm i) \pi_{1} \pi_{3}}+y_{2}(1-i) \sqrt{(1 \mp i) \pi_{2} \pi_{4}}\right] \text { (a) or } \\
\sqrt{\varepsilon_{d}}=\frac{1}{2}\left[y_{1}(1+i) \sqrt{(1 \pm i) \pi_{1} \pi_{4}}+y_{2}(1-i) \sqrt{(1 \mp i) \pi_{2} \pi_{3}}\right] \text { (b). }
\end{gathered}
$$

Moreover the ideal $\mathcal{H}_{0} \mathcal{H}_{1} \mathcal{H}_{3}$ is principal in $\mathbb{k}$, if and only if there exists a unit $\varepsilon \in \mathbb{k}$ such that

$$
(1+i) \pi_{1} \pi_{3} \varepsilon=\alpha^{2},
$$

where $\alpha \in \mathbb{k}$. As $N\left(\varepsilon_{2 p_{1} p_{2}}\right)=-1$, so Lemma 3 involves that $Q_{\mathbb{k}}=1$, the unit index of $\mathbb{k}$; hence $\varepsilon$ is either real or purely imaginary.

Put $\alpha=\alpha_{1}+i \alpha_{2}$, with $\alpha_{1}, \alpha_{2} \in \mathbb{Q}\left(\sqrt{2 p_{1} p_{2}}\right)$, and suppose $\varepsilon$ is real (same proof if it is purely imaginary); as $\pi_{1} \pi_{3}=(e+2 i f)(g+2 i h)=(e g-4 f h)+2 i(e h+g f)$, so the equation (4) is equivalent to

$$
\alpha_{1}^{2}-\alpha_{2}^{2}+2 i \alpha_{1} \alpha_{2}=\varepsilon[(e g-4 f h)-2(e h+f g)]+i \varepsilon_{d}[(e g-4 f h)+2(e h+g f)],
$$

hence

$$
\begin{cases}\alpha_{1}^{2}-\alpha_{2}^{2} & =\varepsilon[(e g-4 f h)-2(e h+f g)] \\ 2 \alpha_{1} \alpha_{2} & =\varepsilon[(e g-4 f h)+2(e h+g f)]\end{cases}
$$


so we get $\alpha_{2}=\frac{\varepsilon[(e g-4 f h)+2(e h+g f)]}{2 \alpha_{1}}$, thus

$$
4 \alpha_{1}^{4}-4 \varepsilon[(e g-4 f h)-2(e h+f g)] \alpha_{1}^{2}-[(e g-4 f h)+2(e h+f g)]^{2} \varepsilon^{2}=0
$$

the discriminant of this equation is $\Delta^{\prime}=4 \varepsilon^{2} d, d=2 p_{1} p_{2}$, which implies that

$$
\alpha_{1}^{2}=\frac{\varepsilon}{4}[2[(e g-4 f h)-2(e h+f g)] \pm 2 \sqrt{d}]
$$

Since

$$
\begin{gathered}
(1+i) \pi_{1} \pi_{3}+(1-i) \pi_{2} \pi_{4}=2(e g-4 f h)-4(e h+f g) \text { and } \\
\sqrt{d}=\sqrt{(1-i) \pi_{1} \pi_{3}} \sqrt{(1+i) \pi_{2} \pi_{4}},
\end{gathered}
$$

then

$$
\begin{aligned}
& \alpha_{1}^{2}=\frac{\varepsilon}{4}\left(\sqrt{(1-i) \pi_{1} \pi_{3}}+\sqrt{(1+i) \pi_{2} \pi_{4}}\right)^{2}, \text { so } \\
& \alpha_{1}=\frac{\sqrt{\varepsilon}}{2}\left(\sqrt{(1-i) \pi_{1} \pi_{3}}+\sqrt{(1+i) \pi_{2} \pi_{4}}\right),
\end{aligned}
$$

therefore if $\varepsilon=\varepsilon_{d}$ and $\sqrt{\varepsilon_{d}}$ takes the value (a), we get

$$
\alpha_{1}=\frac{1}{4}\left(2 y_{1} \pi_{1} \pi_{3}+2 y_{2} \pi_{2} \pi_{4}+\left(y_{1}(1+i)+y_{2}(1-i)\right) \sqrt{d}\right),
$$

and

$$
\alpha_{2}=\frac{\varepsilon_{d}[(e g-4 f h)+2(e h+g f)]}{2 \alpha_{1}},
$$

and it is easy to see that $\alpha_{1}, \alpha_{2} \in \mathbb{Q}\left(\sqrt{2 p_{1} p_{2}}\right)$; hence $\mathcal{H}_{0} \mathcal{H}_{1} \mathcal{H}_{3}$ is principal in $\mathbb{k}$. Proceeding similarly, we prove that $\mathcal{H}_{0} \mathcal{H}_{2} \mathcal{H}_{3}$ is principal in $\mathrm{k}$ if $\sqrt{\varepsilon_{d}}$ takes the value (b). Hence, in $\mathrm{Cl}_{2}(\mathrm{k})$, we have $\mathcal{H}_{3} \sim \mathcal{H}_{0} \mathcal{H}_{1}$ or $\mathcal{H}_{3} \sim \mathcal{H}_{0} \mathcal{H}_{2}$, this in turn shows that, in $\mathrm{Cl}_{2}\left(\mathbb{K}_{3}\right), \mathcal{H}_{1} \mathcal{H}_{3} \sim \mathcal{H}_{0} \mathcal{H}_{1} \mathcal{H}_{2}$ or $\mathcal{H}_{1} \mathcal{H}_{3} \sim \mathcal{H}_{0}$, as we know that $\mathcal{H}_{0}, \mathcal{H}_{1} \mathcal{H}_{2}$ capitulate in $\mathbb{K}_{3}$, so the result. Thus

$$
\mathfrak{A}^{2^{n+1}} \sim 2_{F_{1}}^{2^{n}} \sim 1
$$

Consequently, in $\mathbf{C l}_{2}\left(\mathbb{K}_{3}\right)$, we have

$$
\left\{\begin{array}{l}
\mathfrak{A}^{2^{n+1}} \sim \mathfrak{P}^{2^{m}} \sim \mathcal{H}_{1} \mathcal{H}_{2} \sim 1, \text { if } q=1, \\
\mathfrak{A}^{2^{n+1}} \sim \mathfrak{P}^{2^{m}} \sim \mathcal{H}_{1} \mathcal{H}_{2} \not 1 \text { and } \mathfrak{A}^{2^{n+2}} \sim \mathfrak{P}^{2^{m+1}} \sim 1, \text { if } q=2 .
\end{array}\right.
$$

To this end, note that for all $i \leq n, j \leq m-1$, we have $\mathfrak{A}^{2^{i}} \mathfrak{P}^{2^{j}} \not 1$, as otherwise, we would have, by applying the norm $N_{\mathbb{K}_{3} / F_{1}}, 2_{F_{1}}^{2^{i}} I^{2^{j}} \sim 1$, which contradicts the results of Theorem 4 .

Conclusion 
If $q=1$, then $\langle[\mathfrak{A}],[\mathfrak{P}]\rangle$ is a subgroup of $\mathbf{C} l_{2}\left(\mathbb{K}_{3}\right)$ of type $\left(2^{m}, 2^{n+1}\right)$, and as in this case $h\left(\mathbb{K}_{3}\right)=h\left(p_{1} p_{2}\right) h\left(-p_{1} p_{2}\right)=2^{n+m+1}$ (see Lemma [3), so

$$
\mathbf{C} l_{2}\left(\mathbb{K}_{3}\right)=\langle[\mathfrak{A}],[\mathfrak{P}]\rangle \simeq\left(2^{n+1}, 2^{m}\right) .
$$

If $q=2$, then $\langle[\mathfrak{A}],[\mathfrak{P}]\rangle$ is a subgroup of $\mathbf{C} l_{2}\left(\mathbb{K}_{3}\right)$ of type $\left(2^{\min (m, n+1)}, 2^{\max (n+2, m+1)}\right)$, and as in this case $h\left(\mathbb{K}_{3}\right)=2 h\left(p_{1} p_{2}\right) h\left(-p_{1} p_{2}\right)=$ $2^{n+m+2}$ (see Lemma 31), so

$$
\mathbf{C l} l_{2}\left(\mathbb{K}_{3}\right)=\langle[\mathfrak{A}],[\mathfrak{P}]\rangle \simeq\left(2^{\min (m, n+1)}, 2^{\max (n+2, m+1)}\right)
$$

As $\mathcal{H}_{1}, \mathcal{P}_{1}$ remain inert in $\mathbb{K}_{3}$, so they do not capitulate and coincide in $\mathbb{K}_{3}$, hence they are of order 2. Thus from Theorem 4 and Lemma 5 we deduce that: - If $\left(\frac{p_{1}}{p_{2}}\right)=-1$ or $\left(\frac{p_{1}}{p_{2}}\right)=1$ and $N\left(\varepsilon_{p_{1} p_{2}}\right)=-1$, then

$$
\mathfrak{A}^{2^{n}} \mathfrak{P}^{2^{m-1}} \sim 2_{F_{1}}^{2^{n-1}} I^{2^{m-2}} \sim \mathcal{P}_{1} \sim \mathcal{H}_{1} .
$$

- If $\left(\frac{p_{1}}{p_{2}}\right)=1$ and $N\left(\varepsilon_{p_{1} p_{2}}\right)=1$, then $2_{F_{1}}^{2^{n}} \sim 1$, thus

$$
\mathfrak{P}^{2^{m-1}} \sim I^{2^{m-2}} \sim \mathcal{P}_{1} \sim \mathcal{H}_{1}
$$

Finally, from Lemma 6, we deduce the following remark

Remark 1. (1) Assume $q=1$, so

(i) If $\left(\frac{p_{1}}{p_{2}}\right)=-1$ or $\left(\frac{p_{1}}{p_{2}}\right)=1$ and $\left(\frac{p_{1}}{p_{2}}\right)_{4}\left(\frac{p_{2}}{p_{1}}\right)_{4}=-1$, then $n=1$ and $m \geq 3$, thus $\mathbf{C} l_{2}\left(\mathbb{K}_{3}\right) \simeq\left(4,2^{m}\right)$.

(ii) If $\left(\frac{p_{1}}{p_{2}}\right)=1$ and $\left(\frac{p_{1}}{p_{2}}\right)_{4}\left(\frac{p_{2}}{p_{1}}\right)_{4}=1$, then $m=2$ and $n \geq 2$, hence $\mathbf{C} l_{2}\left(\mathbb{K}_{3}\right) \simeq$ $\left(4,2^{n+1}\right)$.

(2) If $q=2$, then $\mathbf{C l}_{2}\left(\mathbb{K}_{3}\right) \simeq\left(4,2^{n+2}\right)$.

This completes the proof of the second assertion.

(3) For the proof of the third assertion see [4].

(4) Computation of $\operatorname{Gal}\left(\mathbb{k}_{2}^{(2)} / \mathbb{k}\right)$.

Put $L=\mathbb{k}_{2}^{(2)}$, the Hilbert 2-class field of $\mathbb{k}$. Let $\left(\frac{L / \mathbb{K}_{3}}{P}\right)$ denote the Artin symbol for the normal extension $L / \mathbb{K}_{3}$; hence it is clear that $\sigma=\left(\frac{L / \mathbb{K}_{3}}{\mathfrak{P}}\right)$ and $\tau=\left(\frac{L / \mathbb{K}_{3}}{\mathfrak{A}}\right)$ generate the abelian subgroup $\operatorname{Gal}\left(L / \mathbb{K}_{3}\right)$ of $G=\operatorname{Gal}(L / \mathbb{k})$. If we 
put also $\rho=\left(\frac{L / \mathbb{k}}{\mathcal{H}_{1}}\right)$, then $\rho$ restricts to the nontrivial automorphism of $\mathbb{K}_{3} / \mathbb{k}$, since $\mathcal{H}_{1}$ is not norm from $\mathbb{K}_{3} / \mathbb{k}$; from which we deduce that

$$
G=\operatorname{Gal}(L / \mathbb{k})=\langle\rho, \tau, \sigma\rangle .
$$

Note that $|G|=2\left|\operatorname{Gal}\left(L / \mathbb{K}_{3}\right)\right|=\left\{\begin{array}{l}2^{n+m+2} \text { if } q=1, \\ 2^{n+m+3} \text { if } q=2 .\end{array}\right.$

To continue, let us prove the following result.

Lemma 8. In $\mathbf{C} l_{2}(\mathbb{k})$, we have $N_{\mathbb{K}_{3} / \mathbb{k}}(\mathfrak{P}) \sim \mathcal{H}_{1} \mathcal{H}_{2}$.

Proof. We choose a prime ideal $\mathfrak{R}$ in $\mathbb{K}_{3}$ such that $[\mathfrak{R}]=[\mathfrak{P}]$, this is always possible by Chebotarev's theorem, hence $\mathcal{R}_{\mathbb{k}} \sim N_{\mathbb{K}_{3} / \mathbb{k}}(\Re)$ and $\mathcal{R}_{F_{1}} \sim N_{\mathbb{K}_{3} / F_{1}}(\Re)$ are prime ideals in $\mathbb{k}$ and $F_{1}$ respectively, thus $\mathcal{R}_{F_{1}} \sim N_{\mathbb{K}_{3} / F_{1}}(\mathfrak{R}) \sim N_{\mathbb{K}_{3} / F_{1}}(\mathfrak{P}) \sim I$. As the extension $F_{1} / k_{1}$ is ramified and $\mathbf{C l}_{2}\left(k_{1}\right)$ is generated by $2_{1}$, we infer that the prime ideal $\mathcal{R}_{k_{1}} \sim N_{F_{1} / k_{1}}\left(\mathcal{R}_{F_{1}}\right) \sim N_{F_{1} / k_{1}}(I) \sim 2_{1}^{2^{i}}$ with some integer $i$. This implies that $2^{2^{i}} r= \pm\left(x^{2}-p_{1} p_{2} y^{2}\right)$, which in turn shows that $\left(\frac{r}{p_{1}}\right)=1$.

We know that $N_{\mathbb{K}_{3} / \mathbb{k}}\left(\mathbf{C} l_{2}\left(\mathbb{K}_{3}\right)\right)=\left\langle\left[\mathcal{H}_{0}\right],\left[\mathcal{H}_{1} \mathcal{H}_{2}\right]\right\rangle$. So if $N_{\mathbb{K}_{3} / \mathbb{k}}(\mathfrak{P}) \sim \mathcal{H}_{0}$, then $\mathcal{R}_{\mathbb{k}} \sim \mathcal{H}_{0}$ (equivalence in $\mathrm{Cl}_{2}(\mathbb{k})$ ); hence the prime ideal $\mathfrak{r}=N_{\mathbb{k} / k_{0}}\left(\mathcal{R}_{\mathbb{k}}\right)$ of $k_{0}$ is equivalent, in $\mathbf{C} l_{2}\left(k_{0}\right)$, to $\widetilde{2} \sim P_{1} P_{2}$. Therefore the equivalence $\mathfrak{r} \sim \widetilde{2}$ yields that $2 r= \pm\left(x^{2}-2 p_{1} p_{2} y^{2}\right)$, where $x, y$ are in $\mathbb{Z}$; which shows that $\left(\frac{2 r}{p_{1}}\right)=1$, this leads to the contradiction $\left(\frac{r}{p_{1}}\right)=-1$, since $\left(\frac{2}{p_{1}}\right)=-1$. We get the same contradiction if we suppose that $N_{\mathbb{K}_{3} / \mathbb{k}}(\mathfrak{P}) \sim \mathcal{H}_{0} \mathcal{H}_{1} \mathcal{H}_{2}$. Finally the equivalence $N_{\mathbb{K}_{3} / \mathbb{k}}(\mathfrak{P}) \sim 1$ can not occur since the order of $\sigma$ is strictly greater than 1 .

Therefore the following relations hold:

- $[\tau, \sigma]=1$.

- $\rho^{2}=\left(\frac{L / \mathbb{k}_{k}}{\mathcal{H}_{1}^{2}}\right)=\left(\frac{L / \mathbb{k}}{N_{\mathbb{K}_{3} / \mathrm{k}}\left(\mathcal{H}_{1}\right)}\right)=\left(\frac{L / \mathbb{K}_{3}}{\mathcal{H}_{1}}\right)$, so $\rho^{4}=1$.

- $\tau \rho^{-1} \tau \rho=\left(\frac{L / \mathbb{K}_{3}}{\mathfrak{A}^{1+\rho}}\right)=1$, since $\mathfrak{A}^{1+\rho}=N_{\mathbb{K}_{3} / \mathbb{k}}(\mathfrak{A}) \sim \mathcal{H}_{0} \sim 1$, thus $[\tau, \rho]=$ $\tau^{-1} \rho^{-1} \tau \rho=\tau^{-2}$ and $[\rho, \tau]=\tau^{2}$.

- $\sigma \rho^{-1} \sigma \rho=\left(\frac{L / \mathbb{K}_{3}}{\mathfrak{P}^{1+\rho}}\right)=\left\{\begin{array}{l}1, \text { if } q=1, \\ \sigma^{2^{m}}, \text { if } q=2,\end{array}\right.$ since, in $\mathbf{C} l_{2}\left(\mathbb{K}_{3}\right)$, we have $\mathfrak{P}^{1+\rho}=N_{\mathbb{K}_{3} / \mathrm{k}}(\mathfrak{P}) \sim \mathcal{H}_{1} \mathcal{H}_{2} \sim\left\{\begin{array}{l}1, \text { if } q=1, \\ \mathfrak{P}^{2^{m}}, \text { if } q=2,\end{array}\right.$

therefore $[\sigma, \rho]=\left\{\begin{array}{l}\sigma^{-2}, \text { if } q=1, \\ \sigma^{2^{m}-2}=\sigma^{2}, \text { if } q=2, \text { since in this case } m=2 .\end{array}\right.$ 
- Suppose $q=1$, so

- If $\left(\frac{p_{1}}{p_{2}}\right)=-1$ or $\left(\frac{p_{1}}{p_{2}}\right)=1$ and $N\left(\varepsilon_{p_{1} p_{2}}\right)=-1$, then $\rho^{4}=\sigma^{2^{m}}=\tau^{2^{n+1}}=1$ and $\rho^{2}=\sigma^{2^{m-1}} \tau^{2^{n}}$, since $\mathfrak{A}^{2^{n+1}} \sim \mathfrak{P}^{2^{m}} \sim 1$ and $\mathcal{H}_{1} \sim \mathfrak{A}^{2^{n}} \mathfrak{P}^{2^{m-1}}$.

- If $\left(\frac{p_{1}}{p_{2}}\right)=1$ and $N\left(\varepsilon_{p_{1} p_{2}}\right)=1$, then $\rho^{4}=\sigma^{2^{m}}=\tau^{2^{n+1}}=1$ and $\rho^{2}=\sigma^{2^{m-1}}$ since $\mathcal{H}_{1} \sim \mathfrak{P}^{2^{m-1}}$.

- Suppose $q=2$, so necessarily $\left(\frac{p_{1}}{p_{2}}\right)=-1$ or $\left(\frac{p_{1}}{p_{2}}\right)=1$ and $N\left(\varepsilon_{p_{1} p_{2}}\right)=-1$, then $\left\{\begin{array}{l}\rho^{4}=\sigma^{2^{m+1}}=\tau^{2^{n+2}}=1, \\ \sigma^{2^{m}}=\tau^{2^{n+1}} \text { and } \rho^{2}=\sigma^{2^{m-1}} \tau^{2^{n}},\end{array}\right.$ since $\mathfrak{A}^{2^{n+2}} \sim \mathfrak{P}^{2^{m+1}} \sim 1, \mathfrak{A}^{2^{n+1}} \sim \mathfrak{P}^{2^{m}}$ and $\mathcal{H}_{1} \sim \mathfrak{A}^{2^{n}} \mathfrak{P}^{2^{m-1}}$.

(5) As $[\tau, \sigma]=1,[\rho, \sigma]=\sigma^{2}$ or $\sigma^{-2}$ and $[\rho, \tau]=\tau^{2}$, then the derived group of $G$ is $G^{\prime}=\left\langle\sigma^{2}, \tau^{2}\right\rangle$, therefore

$\mathbf{C} l_{2}\left(\mathbb{k}_{2}^{(1)}\right) \simeq\left\{\begin{array}{l}\left(2^{m-1}, 2^{n}\right) \text { if } q=1, \\ \left(2^{\min (m, n+1)-1}, 2^{\max (m+1, n+2)-1}\right)=\left(2,2^{n+1}\right) \text { if } q=2 .\end{array}\right.$

(6) Finally, we compute the coclass of $G$.

Let $G$ be the group defined above. Then the lower central series of $G$ is defined inductively by $\gamma_{1}(G)=G$ and $\gamma_{i+1}(G)=\left[\gamma_{i}(G), G\right]$, that is the subgroup of $G$ generated by the set $\left\{[a, b]=a^{-1} b^{-1} a b / a \in \gamma_{i}(G), b \in G\right\}$, so the coclass of $G$ is defined to be $c c(G)=h-c$, where $|G|=2^{h}$ and $c=c(G)$ is the nilpotency class of $G$, that is the smallest integer satisfying $\gamma_{c+1}(G)=1$. We easily get

$\gamma_{1}(G)=G$.

$\gamma_{2}(G)=G^{\prime}=\left\langle\sigma^{2}, \tau^{2}\right\rangle$.

$\gamma_{3}(G)=\left[G^{\prime}, G\right]=\left\langle\sigma^{4}, \tau^{4}\right\rangle$.

Then Proposition 3(6) (see below) yields that $\gamma_{j+1}(G)=\left[\gamma_{j}(G), G\right]=\left\langle{\sigma^{2}}^{j}, \tau^{2^{j}}\right\rangle$.

Suppose $q=1$, then if we put $v=\max (n, m-1)$, we get $\gamma_{v+2}(G)=\left\langle{\sigma^{2}}^{v+1}, \tau^{2^{v+1}}\right\rangle=\langle 1\rangle$ and $\gamma_{v+1}(G)=\left\langle{\sigma^{2}}^{v}, \tau^{2^{v}}\right\rangle \neq\langle 1\rangle$. As, in this case, $|G|=2^{n+m+2}$, so

$$
c(G)=v+1 \text { and } c c(G)=n+m+1-v=3,
$$

in fact, from Lemma 6, we have $m \geq 3$ and $n=1$ or $m=2$ and $n \geq 2$, so the first case implies that $v=m-1$ and $c c(G)=n+m+1-v=3$, whereas the second one yields that $v=n$ and $c c(G)=n+m+1-v=3$.

Suppose $q=2$, then if we put $v=\max (n+1, m)$, we get $\gamma_{v+2}(G)=\left\langle{\sigma^{2}}^{v+1}, \tau^{2^{v+1}}\right\rangle=\langle 1\rangle$ and $\gamma_{v+1}(G)=\left\langle{\sigma^{2}}^{v}, \tau^{2^{v}}\right\rangle \neq\langle 1\rangle$. As, in this case, 
$|G|=2^{n+m+3}$, so

$$
c(G)=v+1 \text { and } c c(G)=n+m+2-v=3,
$$

since in this case, from Lemma 6, $m=2$ and $n \geq 1$, thus $v=n+1$.

4.2. Proof of Theorem 3. For this we need the following results which are easy to check.

Proposition 3. Let $G=\langle\sigma, \tau, \rho\rangle$ denote the group defined above, then

(1) $\rho^{-1} \sigma \rho=\left\{\begin{array}{l}\sigma^{-1} \text { if } q=1 \\ \sigma^{3} \text { if } q=2\end{array}\right.$

(2) $\rho^{-1} \tau \rho=\tau^{-1}$.

(3) $\left[\rho^{2}, \sigma\right]=\left[\rho^{2}, \tau\right]=1$.

(4) $(\tau \rho)^{2}=\rho^{2}$.

(5) $(\sigma \tau \rho)^{2}=(\sigma \rho)^{2}=\left\{\begin{array}{l}\rho^{2} \text { if } q=1, \\ \rho^{2} \sigma^{4} \text { if } q=2 .\end{array}\right.$

(6) For all $r \in \mathbb{N},\left[\rho, \tau^{2^{r}}\right]=\tau^{2^{r+1}}$ and $\left[\rho, \sigma^{2^{r}}\right]=\left\{\begin{array}{l}\sigma^{2^{r+1}} \text { if } q=1, \\ \sigma^{-2^{r+1}} \text { if } q=2 .\end{array}\right.$

The proof of Theorem 3 consists of 3 parts. In the first part, we will compute $N_{\mathbb{K}_{j} / \mathbb{k}}\left(\mathbf{C} l_{2}\left(\mathbb{K}_{j}\right)\right)$, for all $1 \leq j \leq 7$. In the second one, we will determine the capitulation kernels $\kappa_{\mathbb{K}_{j}}$ and the types of $\mathbf{C l}_{2}\left(\mathbb{K}_{4}\right)$ and in the third one, we will determine the capitulation kernels $\kappa_{\mathbb{L}_{j}}$ and the types of $\mathbf{C l} l_{2}\left(\mathbb{L}_{4}\right)$. It should be noted that if $\left(\frac{p_{1}}{p_{2}}\right)=-1$, then Propositions 1, 2] imply that

$$
\left\{\begin{array}{l}
q=1 \Leftrightarrow\left(\frac{\pi_{1}}{\pi_{3}}\right)=\left(\frac{1+i}{\pi_{1}}\right)\left(\frac{1+i}{\pi_{3}}\right) \\
q=2 \Leftrightarrow\left(\frac{\pi_{1}}{\pi_{3}}\right)=-\left(\frac{1+i}{\pi_{1}}\right)\left(\frac{1+i}{\pi_{3}}\right)
\end{array}\right.
$$

4.2.1. Norm class groups. Let us compute $N_{j}=N_{\mathbb{K}_{j} / \mathbb{k}}\left(\mathbf{C} l_{2}\left(\mathbb{K}_{j}\right)\right)$, the results are summarized in the following table. Note that the left hand sides refer to the case $\left(\frac{\pi_{1}}{\pi_{3}}\right)=-1$, while the right ones refer to the case $\left(\frac{\pi_{1}}{\pi_{3}}\right)=1$. Put $B=\left(\frac{1+i}{\pi_{1}}\right)\left(\frac{1+i}{\pi_{3}}\right)$.

Table 1: Norm class groups

\begin{tabular}{|c|c|c|}
\hline $\mathbb{K}_{j}$ & $N_{j}$ for $\left(\frac{p_{1}}{p_{2}}\right)=1$ & $N_{j}$ for $\left(\frac{p_{1}}{p_{2}}\right)=-1$ \\
\hline $\mathbb{K}_{1}$ & $\left\langle\left[\mathcal{H}_{0} \mathcal{H}_{1}\right],\left[\mathcal{H}_{0} \mathcal{H}_{2}\right]\right\rangle$ & $\left\langle\left[\mathcal{H}_{1}\right],\left[\mathcal{H}_{2}\right]\right\rangle$ \\
\hline $\mathbb{K}_{2}$ & $\left\langle\left[\mathcal{H}_{1}\right],\left[\mathcal{H}_{2}\right]\right\rangle$ & $\left\langle\left[\mathcal{H}_{0} \mathcal{H}_{1}\right],\left[\mathcal{H}_{0} \mathcal{H}_{2}\right]\right\rangle$ \\
\hline $\mathbb{K}_{3}$ & $\left\langle\left[\mathcal{H}_{0}\right],\left[\mathcal{H}_{1} \mathcal{H}_{2}\right]\right\rangle$ & $\left\langle\left[\mathcal{H}_{0}\right],\left[\mathcal{H}_{1} \mathcal{H}_{2}\right]\right\rangle$ \\
\hline
\end{tabular}




\begin{tabular}{|c|c|c|}
\hline $\mathbb{K}_{j}$ & $N_{j}$ for $\left(\frac{p_{1}}{p_{2}}\right)=1$ & \multicolumn{1}{|c|}{$N_{j}$ for $\left(\frac{p_{1}}{p_{2}}\right)=-1$} \\
\hline \multirow{2}{*}{$\mathbb{K}_{4}$} & $\left\langle\left[\mathcal{H}_{0}\right],\left[\mathcal{H}_{2}\right]\right\rangle,\left\langle\left[\mathcal{H}_{0}\right],\left[\mathcal{H}_{1}\right]\right\rangle$ if $B=1$ & $\left\langle\left[\mathcal{H}_{1}\right],\left[\mathcal{H}_{0} \mathcal{H}_{2}\right]\right\rangle\left\langle\left[\mathcal{H}_{0}\right],\left[\mathcal{H}_{2}\right]\right\rangle$ if $q=1$ \\
& $\left\langle\left[\mathcal{H}_{2}\right],\left[\mathcal{H}_{0} \mathcal{H}_{1}\right]\right\rangle,\left\langle\left[\mathcal{H}_{1}\right],\left[\mathcal{H}_{0} \mathcal{H}_{2}\right]\right\rangle$ if $B=-1$ & $\left\langle\left[\mathcal{H}_{0}\right],\left[\mathcal{H}_{1}\right]\right\rangle\left\langle\left[\mathcal{H}_{2}\right],\left[\mathcal{H}_{0} \mathcal{H}_{1}\right]\right\rangle$ if $q=2$ \\
\hline \multirow{2}{*}{$\mathbb{K}_{5}$} & $\left\langle\left[\mathcal{H}_{2}\right],\left[\mathcal{H}_{0} \mathcal{H}_{1}\right]\right\rangle,\left\langle\left[\mathcal{H}_{1}\right],\left[\mathcal{H}_{0} \mathcal{H}_{2}\right]\right\rangle$ if $B=1$ & $\left\langle\left[\mathcal{H}_{0}\right],\left[\mathcal{H}_{2}\right]\right\rangle\left\langle\left[\mathcal{H}_{1}\right],\left[\mathcal{H}_{0} \mathcal{H}_{2}\right]\right\rangle$ if $q=1$ \\
& $\left\langle\left[\mathcal{H}_{0}\right],\left[\mathcal{H}_{2}\right]\right\rangle,\left\langle\left[\mathcal{H}_{0}\right],\left[\mathcal{H}_{1}\right]\right\rangle$ if $B=-1$ & $\left\langle\left[\mathcal{H}_{2}\right],\left[\mathcal{H}_{0} \mathcal{H}_{1}\right]\right\rangle\left\langle\left[\mathcal{H}_{0}\right],\left[\mathcal{H}_{1}\right]\right\rangle$ if $q=2$ \\
\hline \multirow{2}{*}{$\mathbb{K}_{6}$} & $\left\langle\left[\mathcal{H}_{1}\right],\left[\mathcal{H}_{0} \mathcal{H}_{2}\right]\right\rangle,\left\langle\left[\mathcal{H}_{2}\right],\left[\mathcal{H}_{0} \mathcal{H}_{1}\right]\right\rangle$ if $B=1$ & $\left\langle\left[\mathcal{H}_{0}\right],\left[\mathcal{H}_{1}\right]\right\rangle\left\langle\left[\mathcal{H}_{2}\right],\left[\mathcal{H}_{0} \mathcal{H}_{1}\right]\right\rangle$ if $q=1$ \\
& $\left\langle\left[\mathcal{H}_{0}\right],\left[\mathcal{H}_{1}\right]\right\rangle,\left\langle\left[\mathcal{H}_{0}\right],\left[\mathcal{H}_{2}\right]\right\rangle$ if $B=-1$ & $\left\langle\left[\mathcal{H}_{1}\right],\left[\mathcal{H}_{0} \mathcal{H}_{2}\right]\right\rangle\left\langle\left[\mathcal{H}_{0}\right],\left[\mathcal{H}_{2}\right]\right\rangle$ if $q=2$ \\
\hline \multirow{2}{*}{$\mathbb{K}_{7}$} & $\left\langle\left[\mathcal{H}_{0}\right],\left[\mathcal{H}_{1}\right]\right\rangle,\left\langle\left[\mathcal{H}_{0}\right],\left[\mathcal{H}_{2}\right]\right\rangle$ if $B=1$ & $\left\langle\left[\mathcal{H}_{2}\right],\left[\mathcal{H}_{0} \mathcal{H}_{1}\right]\right\rangle\left\langle\left[\mathcal{H}_{0}\right],\left[\mathcal{H}_{1}\right]\right\rangle$ if $q=1$ \\
& $\left\langle\left[\mathcal{H}_{1}\right],\left[\mathcal{H}_{0} \mathcal{H}_{2}\right]\right\rangle,\left\langle\left[\mathcal{H}_{2}\right],\left[\mathcal{H}_{0} \mathcal{H}_{1}\right]\right\rangle$ if $B=-1$ & $\left\langle\left[\mathcal{H}_{0}\right],\left[\mathcal{H}_{2}\right]\right\rangle\left\langle\left[\mathcal{H}_{1}\right],\left[\mathcal{H}_{0} \mathcal{H}_{2}\right]\right\rangle$ if $q=2$ \\
\hline
\end{tabular}

To check the table entries we use Lemmas 2, 7, Propositions 1, 2 and the following results which are easy to prove.

Lemma 9. Let $p_{1} \equiv p_{2} \equiv 1(\bmod 4)$ be primes. Put $p_{1}=\pi_{1} \pi_{2}$ and $p_{2}=\pi_{3} \pi_{4}$, where $\pi_{j} \in \mathbb{Z}[i]$, then

(i) $\left(\frac{\pi_{1}}{\pi_{2}}\right)=\left(\frac{\pi_{3}}{\pi_{4}}\right)=\left\{\begin{array}{l}1 \text { if } p_{1} \equiv p_{2} \equiv 1(\bmod 8), \\ -1 \text { if } p_{1} \equiv p_{2} \equiv 5(\bmod 8)\end{array}\right.$

(ii) If $\left(\frac{p_{1}}{p_{2}}\right)=1$, then $\left(\frac{\pi_{1}}{\pi_{3}}\right)=\left(\frac{\pi_{2}}{\pi_{3}}\right)=\left(\frac{\pi_{1}}{\pi_{4}}\right)=\left(\frac{\pi_{2}}{\pi_{4}}\right)$.

(iii) If $\left(\frac{p_{1}}{p_{2}}\right)=-1$, then $\left(\frac{\pi_{1}}{\pi_{3}}\right)=\left(\frac{\pi_{2}}{\pi_{4}}\right)=-\left(\frac{\pi_{2}}{\pi_{3}}\right)=-\left(\frac{\pi_{1}}{\pi_{4}}\right)$.

(iv) If $\left(\frac{2}{p_{1}}\right)=1$, then $\left(\frac{1+i}{\pi_{1}}\right)=\left(\frac{1+i}{\pi_{2}}\right)$.

(v) If $\left(\frac{2}{p_{1}}\right)=-1$, then $\left(\frac{1+i}{\pi_{1}}\right)=-\left(\frac{1+i}{\pi_{2}}\right)$.

Compute $N_{j}$ in a few cases. Keeping in mind that $\mathcal{H}_{0}, \mathcal{H}_{1}$ and $\mathcal{H}_{2}$ are unramified in $\mathbb{K}_{j} / \mathbb{k}$.

Take first $\mathbb{K}_{1}=\mathbb{k}\left(\sqrt{p_{1}}\right)=\mathbb{k}\left(\sqrt{2 p_{2}}\right)=\mathbb{Q}\left(\sqrt{p_{1}}, \sqrt{2 p_{2}}, i\right)$. As $N_{1}=\left\{[\mathcal{H}] \in \mathbf{C} l_{2}(\mathbb{k}) /\left(\frac{\alpha}{\mathcal{H}}\right)=\right.$ $1\}$, so for $j \in\{1,2\}$ we get

$$
\begin{aligned}
\left(\frac{\mathbb{k}\left(\sqrt{2 p_{2}}\right) / \mathbb{k}}{\mathcal{H}_{j}}\right) & =\left(\frac{\mathbb{k}\left(\sqrt{2 p_{2}}\right) / \mathbb{k}}{\mathcal{H}_{j}}\right)\left(\sqrt{2 p_{2}}\right)\left(\sqrt{2 p_{2}}\right)^{-1} \\
& =\left(\frac{2 p_{2}}{\mathcal{H}_{j}}\right) \\
& =\left(\frac{2 p_{2}}{p_{1}}\right) \\
& =\left(\frac{2}{p_{1}}\right)\left(\frac{p_{1}}{p_{2}}\right) .
\end{aligned}
$$

Thus 
- If $\left(\frac{p_{1}}{p_{2}}\right)=-1$, then $\left[\mathcal{H}_{j}\right] \in N_{1}$, hence $\left.N_{1}=\left\langle\left[\mathcal{H}_{1}\right], \mathcal{H}_{2}\right]\right\rangle$.

- If $\left(\frac{p_{1}}{p_{2}}\right)=1$, then $\left[\mathcal{H}_{j}\right] \notin N_{1}$, hence $\left[\mathcal{H}_{1} \mathcal{H}_{2}\right] \in N_{1}$. Moreover, since $\left(\frac{2}{p_{1}}\right)=-1$, then $\mathcal{H}_{0} \notin N_{1}$; from which we deduce that $\left[\mathcal{H}_{0} \mathcal{H}_{1}\right]$ and $\left[\mathcal{H}_{0} \mathcal{H}_{2}\right]$ are in $N_{1}$, therefore

$$
N_{1}=\left\langle\left[\mathcal{H}_{0} \mathcal{H}_{1}\right],\left[\mathcal{H}_{0} \mathcal{H}_{2}\right]\right\rangle \text {. }
$$

Take an other example, $\mathbb{K}_{4}=\mathbb{k}\left(\sqrt{\pi_{1} \pi_{3}}\right)=\mathbb{k}\left(\sqrt{2 \pi_{2} \pi_{4}}\right)$. First prove that $\left(\frac{\pi_{1} \pi_{3}}{\mathcal{H}_{0}}\right)=$ $\left(\frac{1+i}{\pi_{1}}\right)\left(\frac{1+i}{\pi_{3}}\right)$. As $1+i$ is unramified in both of $\mathbb{Q}\left(\sqrt{\pi_{1} \pi_{3}}\right) / \mathbb{Q}(i)$ and $\mathbb{k} / \mathbb{Q}(i)$, so according to [11, Proposition 4.2, p.112] and Hilbert symbol properties we get

$$
\begin{aligned}
\left(\frac{\pi_{1} \pi_{3}}{\mathcal{H}_{0}}\right)=\left(\frac{\pi_{1} \pi_{3}}{1+i}\right) & =\left(\frac{\pi_{1} \pi_{3}}{1+i}\right)^{v_{1+i}(1+i)} \\
& =\left(\frac{1+i, \pi_{1} \pi_{3}}{1+i}\right) \\
& =\left(\frac{1+i, \pi_{1}}{1+i}\right)\left(\frac{1+i, \pi_{3}}{1+i}\right) .
\end{aligned}
$$

On the other hand, the product formula implies, for $j \in\{1,2\}$, that

$$
\left(\frac{1+i, \pi_{j}}{1+i}\right)\left(\frac{1+i, \pi_{j}}{\pi_{j}}\right) \prod_{\mathcal{P} \neq \pi_{j}, \mathcal{P} \neq 1+i}\left(\frac{1+i, \pi_{j}}{\mathcal{P}}\right)=1 ;
$$

as $\mathcal{P}$ does not divide $\pi_{j}$ and $1+i$, so $\left(\frac{1+i, \pi_{j}}{\mathcal{P}}\right)=1$, which yields that

$$
\left(\frac{1+i, \pi_{j}}{1+i}\right)\left(\frac{1+i, \pi_{j}}{\pi_{j}}\right)=1 \text {, hence }\left(\frac{1+i, \pi_{j}}{1+i}\right)=\left(\frac{1+i, \pi_{j}}{\pi_{j}}\right)=\left(\frac{1+i}{\pi_{j}}\right) \text {. }
$$

This implies the result.

\section{Compute now $N_{4}$.}

We have $\left\{\begin{array}{l}\left(\frac{\pi_{1} \pi_{3}}{\mathcal{H}_{2}}\right)=\left(\frac{\pi_{1} \pi_{3}}{\pi_{2}}\right)=\left(\frac{\pi_{1}}{\pi_{2}}\right)\left(\frac{\pi_{3}}{\pi_{2}}\right)=-\left(\frac{\pi_{2}}{\pi_{3}}\right), \\ \left(\frac{2 \pi_{2} \pi_{4}}{\mathcal{H}_{1}}\right)=\left(\frac{2 \pi_{2} \pi_{4}}{\pi_{1}}\right)=\left(\frac{2}{p_{1}}\right)\left(\frac{\pi_{1}}{\pi_{2}}\right)\left(\frac{\pi_{4}}{\pi_{1}}\right)=\left(\frac{\pi_{1}}{\pi_{4}}\right), \\ \left(\frac{\pi_{1} \pi_{3}}{\mathcal{H}_{0}}\right)=\left(\frac{1+i}{\pi_{1}}\right)\left(\frac{1+i}{\pi_{3}}\right) .\end{array}\right.$

Assume that $\left(\frac{p_{1}}{p_{2}}\right)=1$. So

- If $\left(\frac{p_{1}}{p_{2}}\right)_{4}\left(\frac{p_{2}}{p_{1}}\right)_{4}=\left(\frac{\pi_{1}}{\pi_{3}}\right)=-1$, then $\mathcal{H}_{2} \in N_{4}$ and $\mathcal{H}_{1} \notin N_{4}$;

thus $\left\{\begin{array}{l}\text { If }\left(\frac{1+i}{\pi_{1}}\right)\left(\frac{1+i}{\pi_{3}}\right)=1 \text {, then } N_{4}=\left\langle\left[\mathcal{H}_{0}\right],\left[\mathcal{H}_{2}\right]\right\rangle, \\ \text { If }\left(\frac{1+i}{\pi_{1}}\right)\left(\frac{1+i}{\pi_{3}}\right)=-1 \text {, then } N_{4}=\left\langle\left[\mathcal{H}_{2}\right],\left[\mathcal{H}_{0} \mathcal{H}_{1}\right]\right\rangle .\end{array}\right.$

- If $\left(\frac{p_{1}}{p_{2}}\right)_{4}\left(\frac{p_{2}}{p_{1}}\right)_{4}=\left(\frac{\pi_{1}}{\pi_{3}}\right)=1$, then $\mathcal{H}_{2} \notin N_{4}$ and $\mathcal{H}_{1} \in N_{4}$; 
hence $\left\{\begin{array}{l}\text { If }\left(\frac{1+i}{\pi_{1}}\right)\left(\frac{1+i}{\pi_{3}}\right)=1 \text {, then } N_{4}=\left\langle\left[\mathcal{H}_{0}\right],\left[\mathcal{H}_{1}\right]\right\rangle, \\ \text { If }\left(\frac{1+i}{\pi_{1}}\right)\left(\frac{1+i}{\pi_{3}}\right)=-1 \text {, then } N_{4}=\left\langle\left[\mathcal{H}_{1}\right],\left[\mathcal{H}_{0} \mathcal{H}_{2}\right]\right\rangle .\end{array}\right.$

Assume that $\left(\frac{p_{1}}{p_{2}}\right)=-1$. So

- If $q=1$, then $\left(\frac{\pi_{1}}{\pi_{3}}\right)=\left(\frac{1+i}{\pi_{1}}\right)\left(\frac{1+i}{\pi_{3}}\right)$, hence

$\left\{\begin{array}{l}\text { If }\left(\frac{\pi_{1}}{\pi_{3}}\right)=1, \text { then } N_{4}=\left\langle\left[\mathcal{H}_{0}\right],\left[\mathcal{H}_{2}\right]\right\rangle, \\ \text { If }\left(\frac{\pi_{1}}{\pi_{3}}\right)=-1, \text { then } N_{4}=\left\langle\left[\mathcal{H}_{1}\right],\left[\mathcal{H}_{0} \mathcal{H}_{2}\right]\right\rangle .\end{array}\right.$

- If $q=2$, then $\left(\frac{\pi_{1}}{\pi_{3}}\right)=-\left(\frac{1+i}{\pi_{1}}\right)\left(\frac{1+i}{\pi_{3}}\right)$, hence

$\left\{\begin{array}{l}\text { If }\left(\frac{\pi_{1}}{\pi_{3}}\right)=1 \text {, then } N_{4}=\left\langle\left[\mathcal{H}_{2}\right],\left[\mathcal{H}_{0} \mathcal{H}_{1}\right]\right\rangle, \\ \text { If }\left(\frac{\pi_{1}}{\pi_{3}}\right)=-1, \text { then } N_{4}=\left\langle\left[\mathcal{H}_{0}\right],\left[\mathcal{H}_{1}\right]\right\rangle .\end{array}\right.$

Proceeding similarly, we check the other table inputs.

4.2.2. Capitulation kernels $\kappa_{\mathbb{K}_{j}}$ and $\operatorname{Gal}\left(\mathbb{k}_{2}^{(2)} / \mathbb{K}_{j}\right)$. Let us compute the Galois groups $G_{j}=\operatorname{Gal}\left(\mathbb{k}_{2}^{(2)} / \mathbb{K}_{j}\right)$, the capitulation kernels $\kappa_{\mathbb{K}_{j}}, \kappa_{\mathbb{K}_{j}} \cap N_{j}$ and the types of $\mathbf{C} l_{2}\left(\mathbb{K}_{j}\right)$. The results are summarized in the following tables. Note that the left hand sides refer to the case $\left(\frac{\pi_{1}}{\pi_{3}}\right)=-1$, while the right ones refer to the case $\left(\frac{\pi_{1}}{\pi_{3}}\right)=1$. Put $B=\left(\frac{1+i}{\pi_{1}}\right)\left(\frac{1+i}{\pi_{3}}\right), a=\min (m, n+1)$ and $b=\max (m+1, n+2)$.

Table 2: $\kappa_{\mathbb{K}_{j}}$ for the case $\left(\frac{p_{1}}{p_{2}}\right)=1$.

\begin{tabular}{|c|c|c|c|c|c|}
\hline $\mathbb{K}_{j}$ & & $G_{j}$ & $\kappa_{\mathbb{K}_{j}}$ & $\kappa_{\mathbb{K}_{j}} \cap N_{j}$ & $\mathbf{C} l_{2}\left(\mathbb{K}_{j}\right)$ \\
\hline $\mathbb{K}_{1}$ & & $\left\langle\sigma, \tau \rho, \tau^{2}\right\rangle$ & $\left\langle\left[\mathcal{H}_{1}\right],\left[\mathcal{H}_{2}\right]\right\rangle$ & $\left\langle\left[\mathcal{H}_{1} \mathcal{H}_{2}\right]\right\rangle$ & $(2,2,2)$ \\
\hline $\mathbb{K}_{2}$ & & $\left\langle\sigma, \rho, \tau^{2}\right\rangle$ & $\left\langle\left[\mathcal{H}_{0} \mathcal{H}_{1}\right],\left[\mathcal{H}_{0} \mathcal{H}_{2}\right]\right\rangle$ & $\left\langle\left[\mathcal{H}_{1} \mathcal{H}_{2}\right]\right\rangle$ & $(2,2,2)$ \\
\hline & $\begin{array}{l}q=1 \\
q=2\end{array}$ & $\langle\tau, \sigma\rangle$ & $\begin{array}{c}\left\langle\left[\mathcal{H}_{0}\right],\left[\mathcal{H}_{1} \mathcal{H}_{2}\right]\right\rangle \\
\left\langle\left[\mathcal{H}_{0}\right]\right\rangle\end{array}$ & $\begin{array}{c}N_{3} \\
\left\langle\left[\mathcal{H}_{0}\right]\right\rangle\end{array}$ & $\begin{array}{c}\left(2^{m}, 2^{n+1}\right) \\
\quad\left(2^{a}, 2^{b}\right)\end{array}$ \\
\hline $\mathbb{K}_{4}$ & $\begin{array}{c}B=1 \\
B=-1\end{array}$ & $\begin{array}{c}\left\langle\tau, \rho \sigma, \sigma^{2}\right\rangle\langle\tau, \rho\rangle \\
\left\langle\sigma \tau, \tau \rho, \sigma^{2}\right\rangle\langle\sigma \tau, \rho\rangle\end{array}$ & $\begin{array}{c}\left\langle\left[\mathcal{H}_{0}\right],\left[\mathcal{H}_{1}\right]\right\rangle \\
\left\langle\left[\mathcal{H}_{1}\right],\left[\mathcal{H}_{0} \mathcal{H}_{2}\right]\right\rangle\end{array}$ & $\begin{array}{c}\left\langle\left[\mathcal{H}_{0}\right]\right\rangle \\
\left\langle\left[\mathcal{H}_{0} \mathcal{H}_{1} \mathcal{H}_{2}\right]\right\rangle\end{array}$ & $(2,2,2)(2,4)$ \\
\hline $\mathbb{K}_{5}$ & $\begin{array}{c}B=1 \\
B=-1\end{array}$ & $\begin{array}{c}\left\langle\sigma \tau, \tau \rho, \sigma^{2}\right\rangle\langle\sigma \tau, \rho\rangle \\
\left\langle\tau, \rho \sigma, \sigma^{2}\right\rangle\langle\tau, \rho\rangle\end{array}$ & $\begin{array}{c}\left\langle\left[\mathcal{H}_{1}\right],\left[\mathcal{H}_{0} \mathcal{H}_{2}\right]\right\rangle \\
\left\langle\left[\mathcal{H}_{0}\right],\left[\mathcal{H}_{1}\right]\right\rangle\end{array}$ & $\begin{array}{c}\left\langle\left[\mathcal{H}_{0} \mathcal{H}_{1} \mathcal{H}_{2}\right]\right\rangle \\
\left\langle\left[\mathcal{H}_{0}\right]\right\rangle\end{array}$ & $(2,2,2)(2,4)$ \\
\hline $\mathbb{K}_{6}$ & $\begin{array}{c}B=1 \\
B=-1\end{array}$ & $\begin{array}{c}\left\langle\sigma \tau, \rho, \sigma^{2}\right\rangle\langle\sigma \tau, \tau \rho\rangle \\
\left\langle\tau, \rho, \sigma^{2}\right\rangle\langle\tau, \rho \sigma\rangle\end{array}$ & $\begin{array}{c}\left\langle\left[\mathcal{H}_{2}\right],\left[\mathcal{H}_{0} \mathcal{H}_{1}\right]\right\rangle \\
\left\langle\left[\mathcal{H}_{0}\right],\left[\mathcal{H}_{2}\right]\right\rangle\end{array}$ & $\begin{array}{c}\left\langle\left[\mathcal{H}_{0} \mathcal{H}_{1} \mathcal{H}_{2}\right]\right\rangle \\
\left\langle\left[\mathcal{H}_{0}\right]\right\rangle\end{array}$ & $(2,2,2)(2,4)$ \\
\hline $\mathbb{K}_{7}$ & $\begin{array}{c}B=1 \\
B=-1\end{array}$ & $\begin{array}{c}\left\langle\tau, \rho, \sigma^{2}\right\rangle\langle\tau, \rho \sigma\rangle \\
\left\langle\sigma \tau, \rho, \sigma^{2}\right\rangle\langle\sigma \tau, \tau \rho\rangle\end{array}$ & $\begin{array}{c}\left\langle\left[\mathcal{H}_{0}\right],\left[\mathcal{H}_{2}\right]\right\rangle \\
\left\langle\left[\mathcal{H}_{2}\right],\left[\mathcal{H}_{0} \mathcal{H}_{1}\right]\right\rangle\end{array}$ & $\begin{array}{c}\left\langle\left[\mathcal{H}_{0}\right]\right\rangle \\
\left\langle\left[\mathcal{H}_{0} \mathcal{H}_{1} \mathcal{H}_{2}\right]\right\rangle\end{array} N_{7}$ & $(2,2,2)(2,4)$ \\
\hline
\end{tabular}


Table 3: $\kappa_{\mathbb{K}_{j}}$ for the case $\left(\frac{p_{1}}{p_{2}}\right)=-1$.

\begin{tabular}{|c|c|c|c|c|c|}
\hline $\mathbb{K}_{j}$ & & $G_{j}$ & $\kappa_{\mathbb{K}_{j}}$ & $\kappa_{\mathbb{K}_{j}} \cap N_{j}$ & $\mathbf{C} l_{2}\left(\mathbb{K}_{j}\right)$ \\
\hline $\mathbb{K}_{1}$ & & $\langle\sigma, \rho\rangle$ & $\left\langle\left[\mathcal{H}_{1}\right],\left[\mathcal{H}_{2}\right]\right\rangle$ & $N_{1}$ & $(2,4)$ \\
\hline $\mathbb{K}_{2}$ & & $\langle\sigma, \tau \rho\rangle$ & $\left\langle\left[\mathcal{H}_{0} \mathcal{H}_{1}\right],\left[\mathcal{H}_{0} \mathcal{H}_{2}\right]\right\rangle$ & $N_{2}$ & $(2,4)$ \\
\hline $\mathbb{K}_{3}$ & $\begin{array}{l}q=1 \\
q=2\end{array}$ & $\langle\tau, \sigma\rangle$ & $\begin{array}{c}\left\langle\left[\mathcal{H}_{0}\right],\left[\mathcal{H}_{1} \mathcal{H}_{2}\right]\right\rangle \\
\left\langle\left[\mathcal{H}_{0}\right]\right\rangle\end{array}$ & $\begin{array}{c}N_{3} \\
\left\langle\left[\mathcal{H}_{0}\right]\right\rangle\end{array}$ & $\begin{array}{c}\left(4,2^{m}\right) \\
\left(4,2^{m+1}\right)\end{array}$ \\
\hline $\mathbb{K}_{4}$ & $\begin{array}{l}q=1 \\
q=2\end{array}$ & $\begin{array}{l}\langle\rho, \tau \sigma\rangle\left\langle\tau, \rho \sigma, \sigma^{2}\right\rangle \\
\langle\tau, \rho\rangle\left\langle\sigma \tau, \tau \rho, \sigma^{2}\right\rangle\end{array}$ & $\begin{array}{l}\left\langle\left[\mathcal{H}_{1}\right],\left[\mathcal{H}_{0} \mathcal{H}_{2}\right]\right\rangle\left\langle\left[\mathcal{H}_{0}\right],\left[\mathcal{H}_{1}\right]\right\rangle \\
\left\langle\left[\mathcal{H}_{0}\right],\left[\mathcal{H}_{1}\right]\right\rangle\left\langle\left[\mathcal{H}_{1}\right],\left[\mathcal{H}_{0} \mathcal{H}_{2}\right]\right\rangle\end{array}$ & $\begin{array}{c}N_{4}\left\langle\left[\mathcal{H}_{0}\right]\right\rangle \\
\left\langle\left[\mathcal{H}_{0} \mathcal{H}_{1} \mathcal{H}_{2}\right]\right\rangle\end{array}$ & $(2,4)(2,2,2)$ \\
\hline $\mathbb{K}_{5}$ & $\begin{array}{l}q=1 \\
q=2\end{array}$ & $\begin{array}{l}\left\langle\tau, \rho \sigma, \sigma^{2}\right\rangle\langle\rho, \sigma \tau\rangle \\
\left\langle\sigma \tau, \tau \rho, \sigma^{2}\right\rangle\langle\tau, \rho\rangle\end{array}$ & $\begin{array}{l}\left\langle\left[\mathcal{H}_{0}\right],\left[\mathcal{H}_{1}\right]\right\rangle\left\langle\left[\mathcal{H}_{1}\right],\left[\mathcal{H}_{0} \mathcal{H}_{2}\right]\right\rangle \\
\left\langle\left[\mathcal{H}_{1}\right],\left[\mathcal{H}_{0} \mathcal{H}_{2}\right]\right\rangle\left\langle\left[\mathcal{H}_{0}\right],\left[\mathcal{H}_{1}\right]\right\rangle\end{array}$ & $\begin{array}{c}\left\langle\left[\mathcal{H}_{0}\right]\right\rangle \\
\left\langle\left[\mathcal{H}_{0} \mathcal{H}_{1} \mathcal{H}_{2}\right]\right\rangle\end{array} N_{5}$ & $(2,2,2)(2,4)$ \\
\hline $\mathbb{K}_{6}$ & $\begin{array}{l}q=1 \\
q=2\end{array}$ & $\begin{array}{l}\left\langle\tau, \rho, \sigma^{2}\right\rangle\langle\sigma \tau, \tau \rho\rangle \\
\left\langle\rho, \sigma \tau, \sigma^{2}\right\rangle\langle\tau, \rho \sigma\rangle\end{array}$ & $\begin{array}{l}\left\langle\left[\mathcal{H}_{0}\right],\left[\mathcal{H}_{2}\right]\right\rangle\left\langle\left[\mathcal{H}_{2}\right],\left[\mathcal{H}_{0} \mathcal{H}_{1}\right]\right\rangle \\
\left\langle\left[\mathcal{H}_{2}\right],\left[\mathcal{H}_{0} \mathcal{H}_{1}\right]\right\rangle\left\langle\left[\mathcal{H}_{0}\right],\left[\mathcal{H}_{2}\right]\right\rangle\end{array}$ & $\begin{array}{c}\left\langle\left[\mathcal{H}_{0}\right]\right\rangle \\
\left\langle\left[\mathcal{H}_{0} \mathcal{H}_{1} \mathcal{H}_{2}\right]\right\rangle\end{array} N_{6}$ & $(2,2,2)(2,4)$ \\
\hline $\mathbb{K}_{7}$ & $\begin{array}{l}q=1 \\
q=2\end{array}$ & $\begin{array}{l}\langle\sigma \tau, \tau \rho\rangle\left\langle\rho, \tau, \sigma^{2}\right\rangle \\
\langle\tau, \rho \sigma\rangle\left\langle\rho, \sigma \tau, \sigma^{2}\right\rangle\end{array}$ & $\begin{array}{l}\left\langle\left[\mathcal{H}_{2}\right],\left[\mathcal{H}_{0} \mathcal{H}_{1}\right]\right\rangle\left\langle\left[\mathcal{H}_{0}\right],\left[\mathcal{H}_{2}\right]\right\rangle \\
\left\langle\left[\mathcal{H}_{0}\right],\left[\mathcal{H}_{2}\right]\right\rangle\left\langle\left[\mathcal{H}_{2}\right],\left[\mathcal{H}_{0} \mathcal{H}_{1}\right]\right\rangle\end{array}$ & $\begin{array}{cc} & \left\langle\left[\mathcal{H}_{0}\right]\right\rangle \\
N_{7} & \left\langle\left[\mathcal{H}_{0} \mathcal{H}_{1} \mathcal{H}_{2}\right]\right\rangle\end{array}$ & $(2,4)(2,2,2)$ \\
\hline
\end{tabular}

Before proving these results, note that, from Tables 1, 2 and 3 we get the following remark:

Remark 2. Put $B=\left(\frac{1+i}{\pi_{1}}\right)\left(\frac{1+i}{\pi_{3}}\right)$ and $\pi=\left(\frac{\pi_{1}}{\pi_{3}}\right)$.

$$
\left\{\begin{array}{l}
\kappa_{\mathbb{K}_{1}}=N_{2}, \quad \kappa_{\mathbb{K}_{2}}=N_{1} \text { if }\left(\frac{p_{1}}{p_{2}}\right)=1, \\
\kappa_{\mathbb{K}_{1}}=N_{1}, \quad \kappa_{\mathbb{K}_{2}}=N_{2} \text { if }\left(\frac{p_{1}}{p_{2}}\right)=-1
\end{array}\right.
$$

(2) Assume that $\left(\frac{p_{1}}{p_{2}}\right)=1$, so

- If $\pi=1$, then $\kappa_{\mathbb{R}_{4}}=N_{4}, \kappa_{\mathbb{K}_{5}}=N_{5}, \kappa_{\mathbb{K}_{6}}=N_{6}$ and $\kappa_{\mathbb{K}_{7}}=N_{7}$.

- Else $\quad \kappa_{\mathbb{K}_{4}}=N_{7}, \quad \kappa_{\mathbb{K}_{5}}=N_{6}, \quad \kappa_{\mathbb{K}_{6}}=N_{5} \quad$ and $\quad \kappa_{\mathbb{K}_{7}}=N_{4}$.

(3) Assume that $\left(\frac{p_{1}}{p_{2}}\right)=-1$ and $q=1$, so

$$
\begin{aligned}
& \kappa_{\mathbb{K}_{4}}=\left\{\begin{array}{l}
N_{4} \text { if } \pi=-1, \\
N_{7} \text { if } \pi=1 .
\end{array} \kappa_{\mathbb{K}_{5}}=\left\{\begin{array}{l}
N_{6} \text { if } \pi=-1, \\
N_{5} \text { if } \pi=1 .
\end{array}\right.\right. \\
& \kappa_{\mathbb{K}_{6}}=\left\{\begin{array}{l}
N_{5} \text { if } \pi=-1, \\
N_{6} \text { if } \pi=1 .
\end{array} \kappa_{\mathbb{K}_{7}}=\left\{\begin{array}{l}
N_{7} \text { if } \pi=-1, \\
N_{4} \text { if } \pi=1 .
\end{array}\right.\right.
\end{aligned}
$$

(4) Assume that $\left(\frac{p_{1}}{p_{2}}\right)=-1$ and $q=2$, so

$$
\begin{aligned}
& \kappa_{\mathbb{K}_{4}}=\left\{\begin{array}{l}
N_{4} \text { if } \pi=-1, \\
N_{7} \text { if } \pi=1 .
\end{array} \kappa_{\mathbb{K}_{5}}=\left\{\begin{array}{l}
N_{6} \text { if } \pi=-1, \\
N_{5} \text { if } \pi=1 .
\end{array}\right.\right. \\
& \kappa_{\mathbb{K}_{6}}=\left\{\begin{array}{l}
N_{5} \text { if } \pi=-1, \\
N_{6} \text { if } \pi=1 .
\end{array} \kappa_{\mathbb{K}_{7}}=\left\{\begin{array}{l}
N_{7} \text { if } \pi=-1, \\
N_{4} \text { if } \pi=1 .
\end{array}\right.\right.
\end{aligned}
$$


To check the tables inputs, we use the following relations:

- $\sigma=\left(\frac{L / \mathbb{K}_{3}}{\mathfrak{P}}\right)=\left(\frac{L / \mathbb{k}}{N_{\mathbb{K}_{3} / \mathbb{k}}(\mathfrak{P})}\right)=\left(\frac{L / \mathbb{k}}{\mathcal{H}_{1} \mathcal{H}_{2}}\right)$, since $N_{\mathbb{K}_{3} / \mathbb{k}}(\mathfrak{P}) \sim \mathcal{H}_{1} \mathcal{H}_{2}$.

- $\tau=\left(\frac{L / \mathbb{K}_{3}}{\mathfrak{A}}\right)=\left(\frac{L / \mathbb{k}}{N_{\mathbb{K}_{3} / \mathbb{k}}(\mathfrak{A})}\right)=\left(\frac{L / \mathbb{k}}{\mathcal{H}_{0}}\right)$, because $N_{\mathbb{K}_{3} / \mathbb{k}}(\mathfrak{A}) \sim \mathcal{H}_{0}$.

- $\rho=\left(\frac{L / \mathbb{k}}{\mathcal{H}_{1}}\right)$.

Recall that the Artin map $\phi$ induces the following commutative diagram:

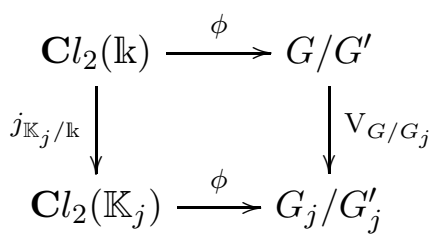

the rows are isomorphisms and $\mathrm{V}_{G / G_{j}}: G / G^{\prime} \longrightarrow G_{j} / G_{j}^{\prime}$ is the group transfer map (Verlagerung) which has the following simple characterization when $G_{j}$ is of index 2 in $G$. Let $G=G_{j} \cup z G_{j}$, then

$$
\mathrm{V}_{G / G_{j}}\left(g G^{\prime}\right)= \begin{cases}g z^{-1} g z \cdot G_{j}^{\prime}=g^{2}[g, z] \cdot G_{j}^{\prime} & \text { if } g \in G_{j} ; \\ g^{2} G_{j}^{\prime} & \text { if } g \notin G_{j} .\end{cases}
$$

Thus $\kappa_{\mathbb{K}_{j}}=\operatorname{ker} j_{\mathbb{K}_{j} / \mathbb{k}}$ is determined by $\operatorname{ker} \mathrm{V}_{G / G_{j}}$.

(a) Consider the extension $\mathbb{K}_{1}$; we know that $G=\langle\sigma, \tau, \rho\rangle$ and, according to the table 1 .

$N_{1}= \begin{cases}\left\langle\left[\mathcal{H}_{0} \mathcal{H}_{1}\right],\left[\mathcal{H}_{0} \mathcal{H}_{2}\right]\right\rangle=\left\langle\left[\mathcal{H}_{1} \mathcal{H}_{2}\right],\left[\mathcal{H}_{0} \mathcal{H}_{1}\right]\right\rangle & \text { if }\left(\frac{p_{1}}{p_{2}}\right)=1, \\ \left\langle\left[\mathcal{H}_{1}\right],\left[\mathcal{H}_{2}\right]\right\rangle=\left\langle\left[\mathcal{H}_{1} \mathcal{H}_{2}\right],\left[\mathcal{H}_{1}\right]\right\rangle & \text { if }\left(\frac{p_{1}}{p_{2}}\right)=-1 .\end{cases}$

Thus $G_{1}=\operatorname{Gal}\left(L / \mathbb{K}_{1}\right)= \begin{cases}\left\langle\sigma, \tau \rho, G^{\prime}\right\rangle=\left\langle\sigma, \tau \rho, \tau^{2}\right\rangle & \text { if }\left(\frac{p_{1}}{p_{2}}\right)=1, \\ \left\langle\sigma, \rho, G^{\prime}\right\rangle=\left\langle\sigma, \rho, \tau^{2}\right\rangle=\langle\sigma, \rho\rangle & \text { if }\left(\frac{p_{1}}{p_{2}}\right)=-1 .\end{cases}$

This implies that $G / G_{1}=\langle\tau\rangle=\left\{1, \tau G_{1}\right\}$; as $[\tau \rho, \sigma]=[\rho, \sigma]=\left\{\begin{array}{ll}\sigma^{2} & \text { if } q=1, \\ \sigma^{-2^{m}+2}=\sigma^{-2} & \text { if } q=2,\end{array}\right.$ and $\left[\tau \rho, \tau^{2}\right]=\tau^{4}$.

So $G_{1}^{\prime}=\left\{\begin{array}{ll}\left\langle\tau^{4}, \sigma^{2}\right\rangle & \text { if }\left(\frac{p_{1}}{p_{2}}\right)=1, \\ \left\langle\sigma^{2}\right\rangle & \text { if }\left(\frac{p_{1}}{p_{2}}\right)=-1 ;\end{array}\right.$ from which we deduce that $\operatorname{Gal}\left(L / \mathbb{K}_{1}\right)=G_{1} / G_{1}^{\prime} \simeq\left\{\begin{array}{ll}(2,2,2) & \text { if }\left(\frac{p_{1}}{p_{2}}\right)=1, \\ (2,4) & \text { if }\left(\frac{p_{1}}{p_{2}}\right)=-1,\end{array} \quad\right.$ since $(\tau \rho)^{2}=\rho^{2} \in G_{1}^{\prime}$.

Compute the kernel of $\mathrm{V}_{G / G_{1}}$. 
* $\mathrm{V}_{G / G_{1}}\left(\sigma G^{\prime}\right)=\sigma^{2}[\sigma, \rho] G_{1}^{\prime}=\sigma^{2} \sigma^{-2} G_{1}^{\prime}$ ou $\sigma^{4} G_{1}^{\prime}=G_{1}^{\prime}$.

$* \mathrm{~V}_{G / G_{1}}\left(\tau G^{\prime}\right)=\tau^{2} G_{1}^{\prime} \neq G_{1}^{\prime}$.

* $\mathrm{V}_{G / G_{1}}\left(\rho G^{\prime}\right)=\rho^{2} G_{1}^{\prime}=G_{1}^{\prime}$.

Consequently

$$
\operatorname{ker} \mathrm{V}_{G / G_{1}}=\left\langle\sigma G^{\prime}, \rho G^{\prime}\right\rangle,
$$

thus

$$
\kappa_{\mathbb{K}_{1}}=\left\langle\left[\mathcal{H}_{1} \mathcal{H}_{2}\right],\left[\mathcal{H}_{1}\right]\right\rangle=\left\langle\left[\mathcal{H}_{1}\right],\left[\mathcal{H}_{2}\right]\right\rangle
$$

(b) For $\mathbb{K}_{2}$, we proceed similarly, we get

$G_{2}=\operatorname{Gal}\left(L / \mathbb{K}_{2}\right)=\left\{\begin{array}{ll}\left\langle\sigma, \rho, \tau^{2}\right\rangle & \text { if }\left(\frac{p_{1}}{p_{2}}\right)=1, \\ \langle\sigma, \tau \rho\rangle & \text { if }\left(\frac{p_{1}}{p_{2}}\right)=-1 ;\end{array}\right.$ this implies that $G_{2}^{\prime}=\left\{\begin{array}{ll}\left\langle\tau^{4}, \sigma^{2}\right\rangle & \text { if }\left(\frac{p_{1}}{p_{2}}\right)=1, \\ \left\langle\sigma^{2}\right\rangle & \text { if }\left(\frac{p_{1}}{p_{2}}\right)=-1 ;\end{array}\right.$ from which we deduce that

$\operatorname{Gal}\left(L / \mathbb{K}_{2}\right)=G_{2} / G_{2}^{\prime} \simeq \begin{cases}(2,2,2) & \text { if }\left(\frac{p_{1}}{p_{2}}\right)=1, \\ (2,4) & \text { if }\left(\frac{p_{1}}{p_{2}}\right)=-1 .\end{cases}$

Thus

$* \mathrm{~V}_{G / G_{2}}\left(\sigma G^{\prime}\right)=\sigma^{2}[\sigma, \rho] G_{2}^{\prime}=\sigma^{2} \sigma^{-2} G_{2}^{\prime}$ or $\sigma^{4} G_{2}^{\prime}=G_{2}^{\prime}$.

$* \mathrm{~V}_{G / G_{2}}\left(\tau G^{\prime}\right)=\tau^{2} G_{2}^{\prime} \neq G_{2}^{\prime}$.

$* \mathrm{~V}_{G / G_{2}}\left(\rho G^{\prime}\right)=\rho^{2}[\rho, \tau] G_{2}^{\prime}=\tau^{2} G_{2}^{\prime} \neq G_{2}^{\prime}$.

* $\mathrm{V}_{G / G_{2}}\left(\tau \rho G^{\prime}\right)=(\tau \rho)^{2} G_{2}^{\prime}=\rho^{2} G_{2}^{\prime}=G_{2}^{\prime}$.

Therefore

$$
\operatorname{ker} \mathrm{V}_{G / G_{2}}=\left\langle\sigma G^{\prime}, \tau \rho G^{\prime}\right\rangle
$$

hence

$$
\kappa_{\mathbb{K}_{2}}=\left\langle\left[\mathcal{H}_{1} \mathcal{H}_{2}\right],\left[\mathcal{H}_{0} \mathcal{H}_{1}\right]\right\rangle=\left\langle\left[\mathcal{H}_{0} \mathcal{H}_{1}\right],\left[\mathcal{H}_{0} \mathcal{H}_{2}\right]\right\rangle .
$$

(c) Take $\mathbb{K}_{4}=\mathbb{k}\left(\sqrt{\pi_{1} \pi_{3}}\right)$ and assume $\left(\frac{p_{1}}{p_{2}}\right)=1$. We have to consider the following two cases:

$1^{\text {st }}$ case: Suppose that $\left(\frac{p_{1}}{p_{2}}\right)_{4}\left(\frac{p_{2}}{p_{1}}\right)_{4}=-1$, then $n=1, m \geq 3, q=1, N\left(\varepsilon_{p_{1} p_{2}}\right)=$ 1 and, from Lemma 2, $\left(\frac{\pi_{1}}{\pi_{3}}\right)=-1$; this in turn has two sub-cases:

$(\alpha)$ If $\left(\frac{1+i}{\pi_{1}}\right)\left(\frac{1+i}{\pi_{3}}\right)=1$, then Table 1 implies that

$$
\begin{gathered}
N_{\mathbb{K}_{4} / \mathbb{k}}\left(\mathbf{C l}_{2}\left(\mathbb{K}_{4}\right)\right)=\left\langle\left[\mathcal{H}_{0}\right],\left[\mathcal{H}_{2}\right]\right\rangle \text {. As }\left(\frac{L / \mathbb{k}}{\mathcal{H}_{2}}\right)=\left(\frac{L / \mathbb{k}}{\mathcal{H}_{1}}\right)^{-1}\left(\frac{L / \mathbb{k}}{\mathcal{H}_{1} \mathcal{H}_{2}}\right)=\rho^{-1} \sigma, \text { so } \\
G_{4}=\operatorname{Gal}\left(L / \mathbb{K}_{4} /\right)=\left\langle\tau, \rho^{-1} \sigma, G^{\prime}\right\rangle .
\end{gathered}
$$


On the other hand, in this sub-case we have $\rho^{-1} \sigma \rho=\sigma^{-1}$, thus $\rho^{-1} \sigma=(\rho \sigma)^{-1}$, therefore

$$
G_{4}=\operatorname{Gal}\left(L / \mathbb{K}_{4} /\right)=\left\langle\tau, \rho \sigma, \sigma^{2}\right\rangle .
$$

Since $\left[\rho \sigma, \sigma^{2}\right]=\sigma^{4},[\rho \sigma, \tau]=\tau^{2}$ and $\left[\sigma, \tau^{2}\right]=1$, so $G_{4}^{\prime}=\left\langle\tau^{2}, \sigma^{4}\right\rangle$. From which we deduce that $G_{4} / G_{4}^{\prime} \simeq(2,2,2)$, since $(\rho \sigma)^{2}=\rho^{2}=\sigma^{2^{m-1}}$. Moreover $G / G_{4}=\langle\sigma\rangle$, then:

$* \mathrm{~V}_{G / G_{4}}\left(\sigma G^{\prime}\right)=\sigma^{2} G_{4}^{\prime} \neq G_{4}^{\prime}$.

$* \mathrm{~V}_{G / G_{4}}\left(\tau G^{\prime}\right)=\tau^{2}[\tau, \sigma] G_{4}^{\prime}=G_{4}^{\prime}$.

* $\mathrm{V}_{G / G_{4}}\left(\rho G^{\prime}\right)=\rho^{2} G_{4}^{\prime}=G_{4}^{\prime}$.

Consequently

$$
k e r \mathrm{~V}_{G / G_{4}}=\left\langle\tau G^{\prime}, \rho G^{\prime}\right\rangle,
$$

and thus

$$
\kappa_{\mathbb{K}_{4}}=\left\langle\left[\mathcal{H}_{0}\right],\left[\mathcal{H}_{1}\right]\right\rangle .
$$

( $\beta)$ If $\left(\frac{1+i}{\pi_{1}}\right)\left(\frac{1+i}{\pi_{3}}\right)=-1$, similarly we get

$$
N_{4}=\left\langle\left[\mathcal{H}_{2}\right],\left[\mathcal{H}_{0} \mathcal{H}_{1}\right]\right\rangle=\left\langle\left[\mathcal{H}_{0} \mathcal{H}_{1} \mathcal{H}_{2}\right],\left[\mathcal{H}_{0} \mathcal{H}_{1}\right]\right\rangle,
$$

thus

$$
G_{4}=\operatorname{Gal}\left(L / \mathbb{K}_{4}\right)=\left\langle\sigma \tau, \tau \rho, \sigma^{2}\right\rangle .
$$

As $[\tau \rho, \sigma \tau]=[\rho, \tau][\rho, \sigma]=(\sigma \tau)^{2}$ and $\left[\tau \rho, \sigma^{2}\right]=\sigma^{4}$, so $G_{4}^{\prime}=\left\langle(\sigma \tau)^{2}\right\rangle$, since $\sigma^{4}=$ $\sigma^{4} \tau^{4}=(\sigma \tau)^{4}$. It is clear that $(\sigma \tau)^{2}, \sigma^{4}$ and $(\tau \rho)^{2}=\rho^{2}=(\tau \sigma)^{2^{m-1}}$ are in $G_{4}^{\prime}$; hence $G_{4} / G_{4}^{\prime} \simeq(2,2,2)$.

Let us compute the kernel of $\mathrm{V}_{G / G_{4}}$. Since $G / G_{4}=\langle\sigma\rangle$, then:

$* \mathrm{~V}_{G / G_{4}}\left(\sigma G^{\prime}\right)=\sigma^{2} G_{4}^{\prime} \neq G_{4}^{\prime}$.

$* \mathrm{~V}_{G / G_{4}}\left(\sigma \tau G^{\prime}\right)=(\sigma \tau)^{2}[\sigma \tau, \sigma] G_{4}^{\prime}=G_{4}^{\prime}$.

$* \mathrm{~V}_{G / G_{4}}\left(\rho G^{\prime}\right)=\rho^{2} G_{4}^{\prime}=G_{4}^{\prime}$.

This implies that

$$
\operatorname{ker} \mathrm{V}_{G / G_{4}}=\left\langle\rho G^{\prime}, \sigma \tau G^{\prime}\right\rangle
$$

hence

$$
\kappa_{\mathbb{K}_{2}}=\left\langle\left[\mathcal{H}_{1}\right],\left[\mathcal{H}_{1} \mathcal{H}_{2} \mathcal{H}_{0}\right]\right\rangle=\left\langle\left[\mathcal{H}_{0} \mathcal{H}_{2}\right],\left[\mathcal{H}_{1}\right]\right\rangle .
$$

- $2^{\text {nd }}$ case: Suppose $\left(\frac{p_{1}}{p_{2}}\right)_{4}\left(\frac{p_{2}}{p_{1}}\right)_{4}=1$, then $m=2, n \geq 2$ and, from Lemma 2 , $\left(\frac{\pi_{1}}{\pi_{3}}\right)=1$. Similarly, there are two sub-cases to distinguish: 
$(\alpha)$ If $\left(\frac{1+i}{\pi_{1}}\right)\left(\frac{1+i}{\pi_{3}}\right)=1$, then the table[implies that $N_{4}=\left\langle\left[\mathcal{H}_{0}\right],\left[\mathcal{H}_{1}\right]\right\rangle$, from which we deduce that

$$
G_{4}=\operatorname{Gal}\left(L / \mathbb{K}_{4} /\right)=\left\langle\tau, \rho, G^{\prime}\right\rangle=\left\langle\tau, \rho, \sigma^{2}\right\rangle ;
$$

as $\rho^{2}=\sigma^{2}$ or $\rho^{2}=\sigma^{2} \tau^{2^{n}}$, so

$$
G_{4}=\operatorname{Gal}\left(L / \mathbb{K}_{4} /\right)=\langle\tau, \rho\rangle .
$$

We know that $[\rho, \tau]=\tau^{2}$, thus $G_{4}^{\prime}=\left\langle\tau^{2}\right\rangle$, this in turn yields that $G_{4} / G_{4}^{\prime} \simeq(2,4)$, since $\rho^{4}=1$. Moreover $G / G_{4}=\langle\sigma\rangle$, so:

$* \mathrm{~V}_{G / G_{4}}\left(\sigma G^{\prime}\right)=\sigma^{2} G_{4}^{\prime} \neq G_{4}^{\prime}$.

$* \mathrm{~V}_{G / G_{4}}\left(\tau G^{\prime}\right)=\tau^{2}[\tau, \sigma] G_{4}^{\prime}=G_{4}^{\prime}$.

$* \mathrm{~V}_{G / G_{4}}\left(\rho G^{\prime}\right)=\rho^{2}[\rho, \sigma] G_{4}^{\prime}=\left\{\begin{array}{l}\rho^{2} \sigma^{2} G_{4}^{\prime}=\sigma^{4} G_{4}^{\prime}=G_{4}^{\prime}, \text { if } q=1, \\ \rho^{2} \sigma^{-2} G_{4}^{\prime}=\sigma^{2} \sigma^{-2} \tau^{2^{n}} G_{4}^{\prime}=G_{4}^{\prime}, \text { if } q=2 ;\end{array}\right.$

Consequently

$$
\operatorname{ker} \mathrm{V}_{G / G_{4}}=\left\langle\tau G^{\prime}, \rho G^{\prime}\right\rangle,
$$

and thus

$$
\kappa_{\mathbb{K}_{4}}=\left\langle\left[\mathcal{H}_{0}\right],\left[\mathcal{H}_{1}\right]\right\rangle .
$$

( $\beta)$ If $\left(\frac{1+i}{\pi_{1}}\right)\left(\frac{1+i}{\pi_{3}}\right)=-1$, then we get

$$
N_{4}=\left\langle\left[\mathcal{H}_{1}\right],\left[\mathcal{H}_{0} \mathcal{H}_{2}\right]\right\rangle=\left\langle\left[\mathcal{H}_{1} \mathcal{H}_{2} \mathcal{H}_{0}\right],\left[\mathcal{H}_{1}\right]\right\rangle,
$$

hence

$$
G_{4}=\operatorname{Gal}\left(L / \mathbb{K}_{4} /\right)=\left\langle\sigma \tau, \rho, \sigma^{2}, \tau^{2}\right\rangle=\langle\sigma \tau, \rho\rangle .
$$

As $[\rho, \sigma \tau]=[\rho, \tau][\rho, \sigma]= \begin{cases}(\sigma \tau)^{2} & \text { if } q=1, \\ \sigma^{-2} \tau^{2} & \text { if } q=2,\end{cases}$

so $G_{4}^{\prime}= \begin{cases}\left\langle(\sigma \tau)^{2}\right\rangle & \text { if } q=1, \\ \left\langle\sigma^{-2} \tau^{2}\right\rangle & \text { if } q=2 .\end{cases}$

Moreover, as $\sigma^{-2} \tau^{2}=\sigma^{-4} \sigma^{2} \tau^{2}=\tau^{-2^{n+1}}(\sigma \tau)^{2}=(\sigma \tau)^{2+2^{n+1}}$, so $G_{4}^{\prime}=\left\langle(\sigma \tau)^{2}\right\rangle$, thus $G_{4} / G_{4}^{\prime} \simeq(2,4)$. On the other hand, $G / G_{4}=\langle\tau\rangle$, hence:

$* \mathrm{~V}_{G / G_{4}}\left(\sigma G^{\prime}\right)=\sigma^{2} G_{4}^{\prime} \neq G_{4}^{\prime}$.

$* \mathrm{~V}_{G / G_{4}}\left(\tau G^{\prime}\right)=\tau^{2} G_{4}^{\prime} \neq G_{4}^{\prime}$.

$* \mathrm{~V}_{G / G_{4}}\left(\rho G^{\prime}\right)=\rho^{2}[\rho, \tau] G_{4}^{\prime}=\rho^{2} \tau^{2} G_{4}^{\prime}=$ $\begin{cases}(\sigma \tau)^{2} G_{4}^{\prime}=G_{4}^{\prime} & \text { if } q=1, \\ \sigma^{2} \tau^{2^{n}} \tau^{2} G_{4}^{\prime}=\sigma^{2} \tau^{-4} \tau^{2} G_{4}^{\prime}=\sigma^{2} \tau^{-2} G_{4}^{\prime}=G_{4}^{\prime} & \text { if } q=2 ;\end{cases}$ since in the second case $(q=2)$ we have $\tau^{2^{n+2}}=1$, thus $\tau^{2^{n}}=\tau^{-4}$. 
* $\mathrm{V}_{G / G_{4}}\left(\sigma \tau G^{\prime}\right)=(\sigma \tau)^{2}[\sigma \tau, \tau] G_{4}^{\prime}=G_{4}^{\prime}$.

Therefore

$$
\operatorname{ker} \mathrm{V}_{G / G_{4}}=\left\langle\rho G^{\prime}, \sigma \tau G^{\prime}\right\rangle
$$

from which we deduce that

$$
\kappa_{\mathbb{K}_{4}}=\left\langle\left[\mathcal{H}_{1}\right],\left[\mathcal{H}_{1} \mathcal{H}_{2} \mathcal{H}_{0}\right]\right\rangle=\left\langle\left[\mathcal{H}_{0} \mathcal{H}_{2}\right],\left[\mathcal{H}_{1}\right]\right\rangle .
$$

Conclusion: Let $\mathbb{K}_{4}=\mathbb{k}_{k}\left(\sqrt{\pi_{1} \pi_{3}}\right)$ and $G_{4}=\operatorname{Gal}\left(L / \mathbb{K}_{4}\right)$. Then $\mathbf{C l}_{2}\left(\mathbb{K}_{4}\right)$ is of type $(2,2,2)$ if $\left(\frac{p_{1}}{p_{2}}\right)_{4}\left(\frac{p_{2}}{p_{1}}\right)_{4}=-1$, and of type $(2,4)$ otherwise. Moreover

(i) If $\left(\frac{1+i}{\pi_{1}}\right)\left(\frac{1+i}{\pi_{3}}\right)=1$, then $\operatorname{ker} \mathrm{V}_{G / G_{4}}=\left\langle\tau G^{\prime}, \rho G^{\prime}\right\rangle$ and $\kappa_{\mathbb{K}_{4}}=\left\langle\left[\mathcal{H}_{0}\right],\left[\mathcal{H}_{1}\right]\right\rangle$.

(ii) If $\left(\frac{1+i}{\pi_{1}}\right)\left(\frac{1+i}{\pi_{3}}\right)=-1$, then $\operatorname{ker} \mathrm{V}_{G / G_{4}}=\left\langle\rho G^{\prime}, \sigma \tau G^{\prime}\right\rangle$, and $\kappa_{\mathbb{K}_{4}}=\left\langle\left[\mathcal{H}_{0} \mathcal{H}_{2}\right],\left[\mathcal{H}_{1}\right]\right\rangle$.

Assume now that $\left(\frac{p_{1}}{p_{2}}\right)=-1$. We have also two cases to distinguish:

$1^{\text {st }}$ case: Suppose $q=1$, so Lemma 6 yields that $n=1, m \geq 3$. Then we need to consider two sub-cases:

- If $\left(\frac{\pi_{1}}{\pi_{3}}\right)=1$, then $\left(\frac{1+i}{\pi_{1}}\right)\left(\frac{1+i}{\pi_{3}}\right)=1$, hence

$$
N_{4}=\left\langle\left[\mathcal{H}_{0}\right],\left[\mathcal{H}_{2}\right]\right\rangle \text { and } G_{4}=\operatorname{Gal}\left(L / \mathbb{K}_{4} /\right)=\left\langle\tau, \rho \sigma, \sigma^{2}\right\rangle
$$

So $G_{4}^{\prime}=\left\langle\tau^{2}, \sigma^{4}\right\rangle$, which involves that $G_{4} / G_{4}^{\prime} \simeq(2,2,2)$, since $(\rho \sigma)^{2}=\rho^{2}=$ $\tau^{2} \sigma^{2^{m-1}}$. Moreover $G / G_{4}=\langle\sigma\rangle$, then:

$* \mathrm{~V}_{G / G_{4}}\left(\sigma G^{\prime}\right)=\sigma^{2} G_{4}^{\prime} \neq G_{4}^{\prime}$.

* $\mathrm{V}_{G / G_{4}}\left(\tau G^{\prime}\right)=\tau^{2}[\tau, \sigma] G_{4}^{\prime}=G_{4}^{\prime}$.

$* \mathrm{~V}_{G / G_{4}}\left(\rho G^{\prime}\right)=\rho^{2} G_{4}^{\prime}=G_{4}^{\prime}$.

Consequently

$$
k e r \mathrm{~V}_{G / G_{4}}=\left\langle\tau G^{\prime}, \rho G^{\prime}\right\rangle
$$

and thus

$$
\kappa_{\mathbb{K}_{2}}=\left\langle\left[\mathcal{H}_{0}\right],\left[\mathcal{H}_{1}\right]\right\rangle .
$$

- If $\left(\frac{\pi_{1}}{\pi_{3}}\right)=-1$, then $\left(\frac{1+i}{\pi_{1}}\right)\left(\frac{1+i}{\pi_{3}}\right)=-1$, similarly we get

$$
N_{4}=\left\langle\left[\mathcal{H}_{1}\right],\left[\mathcal{H}_{0} \mathcal{H}_{2}\right]\right\rangle=\left\langle\left[\mathcal{H}_{0} \mathcal{H}_{1} \mathcal{H}_{2}\right],\left[\mathcal{H}_{1}\right]\right\rangle
$$

thus

$$
G_{4}=\operatorname{Gal}\left(L / \mathbb{K}_{4} /\right)=\langle\sigma \tau, \rho\rangle .
$$

So $G_{4}^{\prime}=\left\langle(\sigma \tau)^{2}\right\rangle$, hence $G_{4} / G_{4}^{\prime} \simeq(2,4)$. As $G / G_{4}=\langle\tau\rangle$, thus

$* \mathrm{~V}_{G / G_{4}}\left(\sigma G^{\prime}\right)=\sigma^{2} G_{4}^{\prime} \neq G_{4}^{\prime}$. 
* $\mathrm{V}_{G / G_{4}}\left(\sigma \tau G^{\prime}\right)=(\sigma \tau)^{2}[\sigma \tau, \sigma] G_{4}^{\prime}=G_{4}^{\prime}$.

* $\mathrm{V}_{G / G_{4}}\left(\rho G^{\prime}\right)=\rho^{2}[\rho, \tau] G_{4}^{\prime}=\rho^{2} \tau^{2} G_{4}^{\prime}=G_{4}^{\prime}$.

This implies that

$$
\operatorname{ker} \mathrm{V}_{G / G_{4}}=\left\langle\rho G^{\prime}, \sigma \tau G^{\prime}\right\rangle
$$

hence

$$
\kappa_{\mathbb{K}_{2}}=\left\langle\left[\mathcal{H}_{1}\right],\left[\mathcal{H}_{1} \mathcal{H}_{2} \mathcal{H}_{0}\right]\right\rangle=\left\langle\left[\mathcal{H}_{0} \mathcal{H}_{2}\right],\left[\mathcal{H}_{1}\right]\right\rangle .
$$

Conclusion. Let $\mathbb{K}_{4}=\mathbb{k}\left(\sqrt{\pi_{1} \pi_{3}}\right)$; assume that $\left(\frac{p_{1}}{p_{2}}\right)=-1$ and $q=1$. If $\left(\frac{\pi_{1}}{\pi_{3}}\right)=-1$, then $\mathbf{C l}_{2}\left(\mathbb{K}_{4}\right)$ is of type $(2,4)$ and of type $(2,2,2)$ otherwise. Moreover

(i) If $\left(\frac{\pi_{1}}{\pi_{3}}\right)=1$, then $\operatorname{ker} \mathrm{V}_{G / G_{4}}=\left\langle\rho G^{\prime}, \tau G^{\prime}\right\rangle$ and $\kappa_{\mathbb{K}_{4}}=\left\langle\left[\mathcal{H}_{0}\right],\left[\mathcal{H}_{1}\right]\right\rangle$.

(ii) If $\left(\frac{\pi_{1}}{\pi_{3}}\right)=-1$, then $\operatorname{ker} \mathrm{V}_{G / G_{4}}=\left\langle\rho G^{\prime}, \sigma \tau G^{\prime}\right\rangle$ and $\kappa_{\mathbb{K}_{4}}=\left\langle\left[\mathcal{H}_{1}\right],\left[\mathcal{H}_{0} \mathcal{H}_{2}\right]\right\rangle$.

$2^{\text {nd }}$ case: Suppose $q=2$, so, according to Lemma $[$, $n=1$ and $m=2$. Then we have two sub-cases to consider :

- If $\left(\frac{\pi_{1}}{\pi_{3}}\right)=1$, then $\left(\frac{1+i}{\pi_{1}}\right)\left(\frac{1+i}{\pi_{3}}\right)=-1$, hence

$$
N_{4}=\left\langle\left[\mathcal{H}_{2}\right],\left[\mathcal{H}_{0} \mathcal{H}_{1}\right]\right\rangle \text { and } G_{4}=\operatorname{Gal}\left(L / \mathbb{K}_{4} /\right)=\left\langle\sigma \tau, \tau \rho, \sigma^{2}\right\rangle \text {. }
$$

So $G_{4}^{\prime}=\left\langle\rho^{2}, \sigma^{4}\right\rangle$, since $\rho^{2}=\tau^{2} \sigma^{2}$; which implies that $G_{4} / G_{4}^{\prime} \simeq(2,2,2)$. Moreover $G / G_{4}=\langle\rho\rangle$, then:

$* \mathrm{~V}_{G / G_{4}}\left(\sigma G^{\prime}\right)=\sigma^{2} G_{4}^{\prime} \neq G_{4}^{\prime}$.

$* \mathrm{~V}_{G / G_{4}}\left(\tau G^{\prime}\right)=\tau^{2} G_{4}^{\prime} \neq G_{4}^{\prime}$.

$* \mathrm{~V}_{G / G_{4}}\left(\rho G^{\prime}\right)=\rho^{2} G_{4}^{\prime}=G_{4}^{\prime}$.

$* \mathrm{~V}_{G / G_{4}}\left(\sigma \tau G^{\prime}\right)=(\sigma \tau)^{2}[\sigma \tau, \rho] G_{4}^{\prime}=(\sigma \tau)^{2}(\sigma \tau)^{-2} \sigma^{4} G_{4}^{\prime}=G_{4}^{\prime}$.

Consequently

$$
\operatorname{ker} \mathrm{V}_{G / G_{4}}=\left\langle\rho G^{\prime}, \sigma \tau G^{\prime}\right\rangle
$$

thus

$$
\kappa_{\mathbb{K}_{2}}=\left\langle\left[\mathcal{H}_{1}\right],\left[\mathcal{H}_{0} \mathcal{H}_{2}\right]\right\rangle .
$$

- If $\left(\frac{\pi_{1}}{\pi_{3}}\right)=-1$, then $\left(\frac{1+i}{\pi_{1}}\right)\left(\frac{1+i}{\pi_{3}}\right)=1$, similarly we get

$$
N_{4}=\left\langle\left[\mathcal{H}_{1}\right],\left[\mathcal{H}_{0}\right]\right\rangle \text { and } G_{4}=\operatorname{Gal}\left(L / \mathbb{K}_{4} /\right)=\langle\tau, \rho\rangle \text {. }
$$

So $G_{4}^{\prime}=\left\langle\tau^{2}\right\rangle$, hence $G_{4} / G_{4}^{\prime} \simeq(2,4)$. As $G / G_{4}=\langle\sigma\rangle$, thus

$* \mathrm{~V}_{G / G_{4}}\left(\sigma G^{\prime}\right)=\sigma^{2} G_{4}^{\prime} \neq G_{4}^{\prime}$.

$* \mathrm{~V}_{G / G_{4}}\left(\tau G^{\prime}\right)=\tau^{2} G_{4}^{\prime}=G_{4}^{\prime}$.

* $\mathrm{V}_{G / G_{4}}\left(\rho G^{\prime}\right)=\rho^{2}[\rho, \sigma] G_{4}^{\prime}=\rho^{2} \sigma^{-2} G_{4}^{\prime}=G_{4}^{\prime}$. 
This implies that

$$
\operatorname{ker} \mathrm{V}_{G / G_{4}}=\left\langle\rho G^{\prime}, \tau G^{\prime}\right\rangle
$$

hence

$$
\kappa_{\mathbb{K}_{2}}=\left\langle\left[\mathcal{H}_{1}\right],\left[\mathcal{H}_{0}\right]\right\rangle \text {. }
$$

Conclusion. Let $\mathbb{K}_{4}=\mathbb{k}\left(\sqrt{\pi_{1} \pi_{3}}\right)$; assume that $\left(\frac{p_{1}}{p_{2}}\right)=-1$ and $q=2$. Then $\mathbf{C} l_{2}\left(\mathbb{K}_{4}\right)$ is of type $(2,4)$ if $\left(\frac{\pi_{1}}{\pi_{3}}\right)=-1$, and of type $(2,2,2)$ otherwise. Moreover

(i) If $\left(\frac{\pi_{1}}{\pi_{3}}\right)=-1$, then $\operatorname{ker} \mathrm{V}_{G / G_{4}}=\left\langle\rho G^{\prime}, \tau G^{\prime}\right\rangle$ and $\kappa_{\mathbb{K}_{4}}=\left\langle\left[\mathcal{H}_{0}\right],\left[\mathcal{H}_{1}\right]\right\rangle$.

(ii) Else $\operatorname{ker} \mathrm{V}_{G / G_{4}}=\left\langle\rho G^{\prime}, \sigma \tau G^{\prime}\right\rangle$ and $\kappa_{\mathbb{K}_{4}}=\left\langle\left[\mathcal{H}_{1}\right],\left[\mathcal{H}_{0} \mathcal{H}_{2}\right]\right\rangle$.

Proceeding similarly we show the other tables inputs.

4.2.3. Capitulation kernels $\kappa_{\mathbb{L}_{j}}$ and $\operatorname{Gal}\left(\mathbb{k}_{2}^{(2)} / \mathbb{L}_{j}\right)$. From the subsection 4.2.2, we deduce that $\kappa_{\mathbb{L}_{j}}=\mathbf{C l}_{2}\left(\mathbb{k}_{k}\right)$. In what follows, we compute the Galois groups $\mathcal{G}_{j}=\operatorname{Gal}\left(\mathbb{k}_{2}^{(2)} / \mathbb{L}_{j}\right)$, their derived groups $\mathcal{G}_{j}^{\prime}$ and the abelian type invariants of $\mathbf{C} l_{2}\left(\mathbb{L}_{j}\right)$. The results are summarized in the following tables; note that the left hand side of columns (if it exists) refers to the case $\left(\frac{1+i}{\pi_{1}}\right)\left(\frac{1+i}{\pi_{3}}\right)=1$, the right hand side to the case $\left(\frac{1+i}{\pi_{1}}\right)\left(\frac{1+i}{\pi_{3}}\right)=-1$.

Put $\left\{\begin{array}{l}\alpha=\min (n, m) \text { and } \beta=\max (n+1, m+1), \\ a=\min (n, m-1) \text { and } b=\max (n+1, m), \\ \pi=\left(\frac{\pi_{1}}{\pi_{3}}\right), \\ B=\left(\frac{1+i}{\pi_{1}}\right)\left(\frac{1+i}{\pi_{3}}\right) .\end{array}\right.$

\begin{tabular}{|c|c|c|c|c|}
\hline $\mathbb{L}_{j}$ & & $\mathcal{G}_{j}$ & $\mathcal{G}_{j}^{\prime}$ & $\mathbf{C} l_{2}\left(\mathbb{L}_{j}\right)$ \\
\hline $\mathbb{L}_{1}$ & & $\left\langle\tau^{2}, \sigma\right\rangle$ & $\langle 1\rangle$ & $\begin{array}{l}\left(2^{n}, 2^{m}\right) \text { if } q=1 \\
\left(2^{\alpha}, 2^{\beta}\right) \text { if } q=2\end{array}$ \\
\hline & $\begin{array}{c}\pi=-1 \\
\pi=1\end{array}$ & $\begin{array}{c}\left\langle\sigma \tau \rho, \sigma^{2}, \tau^{2}\right\rangle\left\langle\tau \rho, \sigma^{2}, \tau^{2}\right\rangle \\
\left\langle\tau \rho, \tau^{2}\right\rangle\left\langle\sigma \tau \rho, \tau^{2}\right\rangle\end{array}$ & $\begin{array}{c}\left\langle\sigma^{4}, \tau^{4}\right\rangle \\
\left\langle\tau^{4}\right\rangle\end{array}$ & $\begin{array}{c}(2,2,2) \\
(2,4)\end{array}$ \\
\hline $\mathbb{L}_{3}$ & $\begin{array}{c}\pi=-1 \\
\pi=1\end{array}$ & $\begin{array}{c}\left\langle\tau \rho, \sigma^{2}, \tau^{2}\right\rangle\left\langle\sigma \tau \rho, \sigma^{2}, \tau^{2}\right\rangle \\
\left\langle\sigma \tau \rho, \tau^{2}\right\rangle\left\langle\tau \rho, \tau^{2}\right\rangle\end{array}$ & $\begin{array}{c}\left\langle\sigma^{4}, \tau^{4}\right\rangle \\
\left\langle\tau^{4}\right\rangle\end{array}$ & $\begin{array}{c}(2,2,2) \\
(2,4)\end{array}$ \\
\hline & $\begin{array}{c}\pi=-1 \\
\pi=1\end{array}$ & $\begin{array}{c}\left\langle\sigma \rho, \sigma^{2}, \tau^{2}\right\rangle \\
\left\langle\rho, \tau^{2}\right\rangle\end{array}$ & $\begin{array}{c}\left\langle\sigma^{4}, \tau^{4}\right\rangle \\
\left\langle\tau^{4}\right\rangle\end{array}$ & $\begin{array}{c}(2,2,2) \\
(2,4)\end{array}$ \\
\hline $\mathbb{L}_{5}$ & $\begin{array}{c}\pi=-1 \\
\pi=1\end{array}$ & $\begin{array}{c}\left\langle\rho, \sigma^{2}, \tau^{2}\right\rangle \\
\left\langle\rho \sigma, \tau^{2}\right\rangle\end{array}$ & $\begin{array}{c}\left\langle\sigma^{4}, \tau^{4}\right\rangle \\
\left\langle\tau^{4}\right\rangle\end{array}$ & $\begin{array}{c}(2,2,2) \\
(2,4)\end{array}$ \\
\hline $\mathbb{L}_{6}$ & $\begin{array}{c}B=-1 \\
B=1\end{array}$ & $\begin{array}{l}\left\langle\sigma \tau, \sigma^{2}\right\rangle \\
\left\langle\tau, \sigma^{2}\right\rangle\end{array}$ & $\begin{array}{l}\langle 1\rangle \\
\langle 1\rangle\end{array}$ & 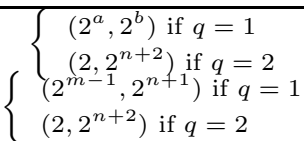 \\
\hline
\end{tabular}

Table 4: Invariants of $\mathbf{C} l_{2}\left(\mathbb{L}_{j}\right)$ for the case $\left(\frac{p_{1}}{p_{2}}\right)=1$ 


\begin{tabular}{|c|c|c|c|}
\hline $\mathbb{L}_{j}$ & $\mathcal{G}_{j}$ & $\mathcal{G}_{j}$ & $\mathbf{C} l_{2}\left(\mathbb{L}_{j}\right)$ \\
\hline $\begin{array}{c}B=-1 \\
\mathbb{L}_{7} \quad B=1\end{array}$ & $\begin{array}{c}\left\langle\tau, \sigma^{2}\right\rangle \\
\left\langle\sigma \tau, \sigma^{2}\right\rangle\end{array}$ & $\begin{array}{l}\langle 1\rangle \\
\langle 1\rangle\end{array}$ & $\begin{array}{l}\left(2^{m-1}, 2^{n+1}\right) \text { if } q=1 \\
\left\{\begin{array}{c}\left(2,2^{n+2}\right) \text { if } q=2 \\
\left(2^{a}, 2^{b}\right) \text { if } q=1 \\
\left(2,2^{n+2}\right) \text { if } q=2\end{array}\right.\end{array}$ \\
\hline
\end{tabular}

For the following table, the left hand side of columns (if it exists) refers to the case $\left(\frac{\pi_{1}}{\pi_{3}}\right)=-1$, the right hand side refers to the case $\left(\frac{\pi_{1}}{\pi_{2}}\right)=1$. Recall that if $\left(\frac{p_{1}}{p_{2}}\right)=-1$, then $n=1$ and $\begin{cases}m=2 & \text { if } q=2, \\ m \geq 2 & \text { if } q=1\end{cases}$

Table 5: Invariants of $\mathbf{C} l_{2}\left(\mathbb{L}_{j}\right)$ for the case $\left(\frac{p_{1}}{p_{2}}\right)=-1$

\begin{tabular}{|c|c|c|c|c|}
\hline $\mathbb{L}_{j}$ & & $\mathcal{G}_{j}$ & $\mathcal{G}_{j}^{\prime}$ & $\mathbf{C} l_{2}\left(\mathbb{L}_{j}\right)$ \\
\hline $\mathbb{L}_{1}$ & & $\left\langle\tau^{2}, \sigma\right\rangle$ & $\langle 1\rangle$ & $\begin{array}{c}\left(2^{n}, 2^{m}\right) \text { if } q=1 \\
\left(2^{n}, 2^{m+1}\right) \text { if } q=2\end{array}$ \\
\hline $\mathbb{L}_{2}$ & & $\left\langle\rho, \sigma^{2}\right\rangle\left\langle\rho \sigma, \sigma^{2}\right\rangle$ & $\left\langle\sigma^{4}\right\rangle$ & $(2,4)$ \\
\hline $\mathbb{L}_{3}$ & & $\left\langle\rho \sigma, \sigma^{2}\right\rangle\left\langle\rho, \sigma^{2}\right\rangle$ & $\left\langle\sigma^{4}\right\rangle$ & $(2,4)$ \\
\hline & $\begin{array}{l}q=1 \\
q=2\end{array}$ & $\begin{array}{c}\left\langle\sigma \tau \rho, \sigma^{2}\right\rangle \\
\left\langle\tau \rho, \sigma^{2}\right\rangle \\
\end{array}$ & $\left\langle\sigma^{4}\right\rangle$ & $(2,4)$ \\
\hline $\mathbb{L}_{5}$ & $\begin{aligned} q & =1 \\
q & =2\end{aligned}$ & $\begin{array}{c}\left\langle\tau \rho, \sigma^{2}\right\rangle \\
\left\langle\sigma \tau \rho, \sigma^{2}\right\rangle\end{array}$ & $\left\langle\sigma^{4}\right\rangle$ & $(2,4)$ \\
\hline $\mathbb{L}_{6}$ & $\begin{array}{l}q=1 \\
q=2\end{array}$ & $\begin{array}{l}\left\langle\sigma \tau, \tau^{2}\right\rangle\left\langle\tau, \sigma^{2}\right\rangle \\
\left\langle\tau, \sigma^{2}\right\rangle\left\langle\sigma \tau, \sigma^{2}\right\rangle\end{array}$ & $\begin{array}{l}\langle 1\rangle \\
\langle 1\rangle\end{array}$ & $\begin{array}{c}\left(2,2^{m}\right)\left(4,2^{m-1}\right) \\
(2,8)(4,4)\end{array}$ \\
\hline $\mathbb{L}_{7}$ & $\begin{aligned} q & =1 \\
q & =2\end{aligned}$ & $\begin{array}{l}\left\langle\tau, \sigma^{2}\right\rangle\left\langle\sigma \tau, \sigma^{2}\right\rangle \\
\left\langle\sigma \tau, \sigma^{2}\right\rangle\left\langle\tau, \sigma^{2}\right\rangle\end{array}$ & $\begin{array}{l}\langle 1\rangle \\
\langle 1\rangle\end{array}$ & $\begin{array}{c}\left(4,2^{m-1}\right)\left(2,2^{m}\right) \\
(4,4)(2,8)\end{array}$ \\
\hline
\end{tabular}

Check the entries in some cases.

$*$ Take $\mathbb{L}_{1}=\mathbb{k}^{(*)}=\mathbb{K}_{1} \cdot \mathbb{K}_{2} \cdot \mathbb{K}_{3}$. Since $\operatorname{Gal}\left(L / \mathbb{L}_{1}\right)=\mathcal{G}_{1}=G_{1} \cap G_{2}$, then $\mathcal{G}_{1}=\left\langle\sigma, \tau \rho, \tau^{2}\right\rangle \cap\left\langle\sigma, \rho, \tau^{2}\right\rangle=\left\langle\sigma, \tau^{2}\right\rangle$, thus $\mathcal{G}_{1}^{\prime}=\langle 1\rangle$. As

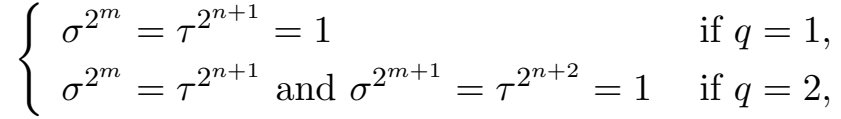
so $\mathbf{C l}_{2}\left(\mathbb{L}_{1}\right) \simeq \begin{cases}\left(2^{n}, 2^{m}\right) & \text { if } q=1, \\ \left(2^{\min (n, m)}, 2^{\max (n+1, m+1)}\right) & \text { if } q=2 .\end{cases}$

* Take $\mathbb{L}_{2}=\mathbb{K}_{1} \cdot \mathbb{K}_{4} \cdot \mathbb{K}_{6}$ and assume that $\left(\frac{p_{1}}{p_{2}}\right)=1$, then $\mathcal{G}_{2}=\operatorname{Gal}\left(L / \mathbb{L}_{2}\right)=$ $G_{1} \cap G_{4} \cap G_{6}$. There are two cases to distinguish:

- $1^{\text {st }}$ case: If $\left(\frac{p_{1}}{p_{2}}\right)_{4}\left(\frac{p_{2}}{p_{1}}\right)_{4}=\left(\frac{\pi_{1}}{\pi_{3}}\right)=-1$, then $q=1$ and $\mathcal{G}_{2}= \begin{cases}\left\langle\sigma, \tau \rho, \tau^{2}\right\rangle \cap\left\langle\sigma \tau, \rho, \tau^{2}\right\rangle=\left\langle\sigma \tau \rho, \sigma^{2}, \tau^{2}\right\rangle & \text { if }\left(\frac{1+i}{\pi_{1}}\right)\left(\frac{1+i}{\pi_{3}}\right)=1, \\ \left\langle\sigma, \tau \rho, \tau^{2}\right\rangle \cap\left\langle\sigma \tau, \tau \rho, \sigma^{2}\right\rangle=\left\langle\tau \rho, \sigma^{2}, \tau^{2}\right\rangle & \text { if }\left(\frac{1+i}{\pi_{1}}\right)\left(\frac{1+i}{\pi_{3}}\right)=-1,\end{cases}$ thus $\mathcal{G}_{2}^{\prime}=\left\langle\sigma^{4}, \tau^{4}\right\rangle$. On the other hand, as in this case $(\sigma \tau \rho)^{2}=(\tau \rho)^{2}=\rho^{2}=\sigma^{2^{m-1}}$, so $\mathrm{Cl}_{2}\left(\mathbb{L}_{2}\right) \simeq(2,2,2)$. 
- $2^{\text {nd }}$ case: If $\left(\frac{p_{1}}{p_{2}}\right)_{4}\left(\frac{p_{2}}{p_{1}}\right)_{4}=\left(\frac{\pi_{1}}{\pi_{3}}\right)=1$, then

$\mathcal{G}_{2}= \begin{cases}\left\langle\sigma, \tau \rho, \tau^{2}\right\rangle \cap\left\langle\tau, \rho, \sigma^{2}\right\rangle=\left\langle\tau \rho, \sigma^{2}, \tau^{2}\right\rangle & \text { if }\left(\frac{1+i}{\pi_{1}}\right)\left(\frac{1+i}{\pi_{3}}\right)=1, \\ \left\langle\sigma, \tau \rho, \tau^{2}\right\rangle \cap\left\langle\sigma \tau, \rho, \sigma^{2}\right\rangle=\left\langle\sigma \tau \rho, \sigma^{2}, \tau^{2}\right\rangle & \text { if }\left(\frac{1+i}{\pi_{1}}\right)\left(\frac{1+i}{\pi_{3}}\right)=-1,\end{cases}$

as, in this case, $(\tau \rho)^{2}=\rho^{2}$ and $(\sigma \tau \rho)^{2}= \begin{cases}\rho^{2}=\sigma^{2} & \text { if } q=1, \\ \rho^{2} \sigma^{4}=\sigma^{2} \tau^{-2^{n}} & \text { if } q=2 ;\end{cases}$

since if $q=2$, we have $\sigma^{4}=\tau^{2^{n+1}}$ and $\rho^{2}=\sigma^{2} \tau^{2^{n}}$,

so $\mathcal{G}_{2}= \begin{cases}\left\langle\tau \rho, \tau^{2}\right\rangle & \text { if }\left(\frac{1+i}{\pi_{1}}\right)\left(\frac{1+i}{\pi_{3}}\right)=1, \\ \left\langle\sigma \tau \rho, \tau^{2}\right\rangle & \text { if }\left(\frac{1+i}{\pi_{1}}\right)\left(\frac{1+i}{\pi_{3}}\right)=-1,\end{cases}$

we infer that $\mathcal{G}_{2}^{\prime}=\left\langle\tau^{4}\right\rangle$. From which we deduce that $\mathbf{C l}_{2}\left(\mathbb{L}_{2}\right) \simeq(2,4)$, since $(\sigma \tau \rho)^{4}=(\tau \rho)^{4}=1$.

Assume now that $\left(\frac{p_{1}}{p_{2}}\right)=-1$, then $\mathcal{G}_{2}= \begin{cases}\left\langle\rho, \sigma^{2}\right\rangle & \text { if }\left(\frac{\pi_{1}}{\pi_{3}}\right)=-1, \\ \left\langle\rho \sigma, \sigma^{2}\right\rangle & \text { if }\left(\frac{\pi_{1}}{\pi_{3}}\right)=1 .\end{cases}$

Thus $\mathcal{G}_{2}^{\prime}=\left\langle\sigma^{4}\right\rangle$, hence $\mathbf{C} l_{2}\left(\mathbb{L}_{2}\right) \simeq(2,4)$.

$*$ Finally, we take $\mathbb{L}_{6}=\mathbb{K}_{3} \cdot \mathbb{K}_{4} \cdot \mathbb{K}_{7}$ and we assume that $\left(\frac{p_{1}}{p_{2}}\right)=1$, then $\operatorname{Gal}\left(L / \mathbb{L}_{6}\right)=$ $\mathcal{G}_{6}=G_{3} \cap G_{4} \cap G_{7}$, which yields that

$\mathcal{G}_{6}= \begin{cases}\langle\tau, \sigma\rangle \cap\left\langle\tau, \rho, \sigma^{2}\right\rangle=\left\langle\tau, \sigma^{2}\right\rangle & \text { if }\left(\frac{1+i}{\pi_{1}}\right)\left(\frac{1+i}{\pi_{3}}\right)=1, \\ \langle\tau, \sigma\rangle \cap\left\langle\sigma \tau, \rho, \sigma^{2}\right\rangle=\left\langle\sigma \tau, \sigma^{2}\right\rangle=\left\langle\sigma \tau, \tau^{2}\right\rangle & \text { if }\left(\frac{1+i}{\pi_{1}}\right)\left(\frac{1+i}{\pi_{3}}\right)=-1,\end{cases}$

therefore $\mathcal{G}_{6}^{\prime}=\langle 1\rangle$.

As $\left\{\begin{array}{ll}\sigma^{2^{m}}=\tau^{2^{n+1}}=1 & \text { if } q=1, \\ \sigma^{2^{m}}=\tau^{2^{n+1}} \text { and } \sigma^{2^{m+1}}=\tau^{2^{n+2}}=1 & \text { if } q=2,\end{array}\right.$ so

- $1^{\text {st }}$ case: If $q=1$, then $\mathrm{Cl}_{2}\left(\mathbb{L}_{6}\right)$ is of type

$\begin{cases}\left(2^{\min (n+1, m-1)}, 2^{\max (n+1, m-1)}\right)=\left(2^{n+1}, 2^{m-1}\right) & \text { if }\left(\frac{1+i}{\pi_{1}}\right)\left(\frac{1+i}{\pi_{3}}\right)=1, \\ \left(2^{\min (n, m-1)}, 2^{\max (n+1, m)}\right) & \text { if }\left(\frac{1+i}{\pi_{1}}\right)\left(\frac{1+i}{\pi_{3}}\right)=-1,\end{cases}$

- $2^{\text {nd }}$ case: If $q=2$, then $m=2, n \geq 2$ and $\mathbf{C} l_{2}\left(\mathbb{L}_{6}\right)$ is of type

$\begin{cases}\left(2^{\min (n+1, m-1)}, 2^{\max (n+2, m+1)}\right)=\left(2,2^{n+2}\right) & \text { if }\left(\frac{1+i}{\pi_{1}}\right)\left(\frac{1+i}{\pi_{3}}\right)=1, \\ \left(2^{\min (n, m-1)}, 2^{\max (n+2, m+1)}\right)=\left(2,2^{n+2}\right) & \text { if }\left(\frac{1+i}{\pi_{1}}\right)\left(\frac{1+i}{\pi_{3}}\right)=-1,\end{cases}$

Assume that $\left(\frac{p_{1}}{p_{2}}\right)=-1$, so

- $1^{\text {st }}$ case: If $q=1$, then $\mathcal{G}_{6}= \begin{cases}\left\langle\tau \sigma, \tau^{2}\right\rangle & \text { if }\left(\frac{\pi_{1}}{\pi_{3}}\right)=-1, \\ \left\langle\tau, \sigma^{2}\right\rangle & \text { if }\left(\frac{\pi_{1}}{\pi_{3}}\right)=1,\end{cases}$

therefore $\mathcal{G}_{6}^{\prime}=\langle 1\rangle$. So $\mathbf{C} l_{2}\left(\mathbb{L}_{6}\right) \simeq \begin{cases}\left(2,2^{m}\right) & \text { if }\left(\frac{\pi_{1}}{\pi_{3}}\right)=-1, \\ \left(4,2^{m-1}\right) & \text { if }\left(\frac{\pi_{1}}{\pi_{3}}\right)=1,\end{cases}$

- $2^{\text {nd }}$ case: If $q=2$, then $m=2, n=1$ and $\mathcal{G}_{6}= \begin{cases}\left\langle\tau, \sigma^{2}\right\rangle & \text { if }\left(\frac{\pi_{1}}{\pi_{3}}\right)=-1 \\ \left\langle\tau \sigma, \tau^{2}\right\rangle & \text { if }\left(\frac{\pi_{1}}{\pi_{3}}\right)=1\end{cases}$ 
Hence $\mathbf{C l}_{2}\left(\mathbb{L}_{6}\right) \simeq \begin{cases}(2,8)=\left(2,2^{n+2}\right) & \text { if }\left(\frac{\pi_{1}}{\pi_{3}}\right)=-1, \\ (4,4) & \text { if }\left(\frac{\pi_{1}}{\pi_{3}}\right)=1,\end{cases}$

The other tables entries are checked similarly.

\section{NUMERICAL EXAMPLES}

Table 6 gives the structure of the class group $\mathbf{C l}(\mathrm{k})$ of the bicyclic biquadratic field $\mathbb{k}=\mathbb{Q}\left(\sqrt{2 p_{1} p_{2}}, i\right)$, its discriminant $\operatorname{disc}(\mathbb{k})$, the structures of the class groups of its two quadratic subfields $k_{0}, \bar{k}_{0}$ and the coclass of $G=\operatorname{Gal}\left(\mathbb{k}_{2}^{(2)} / \mathbb{k}\right)$. Tables 7 and 8 give the structures of the class groups $\mathbf{C l}\left(\mathbb{K}_{j}\right)$. Tables 9 and 10 give the structures of the class groups $\mathbf{C l}\left(\mathbb{L}_{j}\right)$. Finally, Tables $[11$ and 12 give the structures of the class groups of $\mathbf{C l}\left(\mathbb{K}_{j}\right)$ and $\mathbf{C l}\left(\mathbb{L}_{j}\right)$ for the case $\left(\frac{p_{1}}{p_{2}}\right)=-1$. Note that $\pi=\left(\frac{\pi_{1}}{\pi_{3}}\right)$ and $b=\left(\frac{1+i}{\pi_{1}}\right)\left(\frac{1+i}{\pi_{3}}\right)$. Computation are made using PARI/GP [18].

Table 6: Invariants of $\mathbb{k}$

\begin{tabular}{|c|c|c|c|c|c|c|c|c|}
\hline$d=p_{1} \cdot p_{2} \cdot q$ & $q$ & $\left(\frac{p_{1}}{p_{2}}\right)$ & $m, n$ & $\mathbf{C} l_{2}\left(k_{0}\right)$ & $\mathbf{C} l_{2}\left(\bar{k}_{0}\right)$ & $\mathbf{C} l_{2}(\mathbb{k})$ & $\operatorname{disc}(\mathbb{k})$ & $c c(G)$ \\
\hline $130=2.5 .13$ & 2 & -1 & 2,1 & $(2,2)$ & $(2,2)$ & $(2,2,2)$ & 1081600 & 3 \\
$290=2.5 .29$ & 1 & 1 & 2,2 & $(2,2)$ & $(10,2)$ & $(10,2,2)$ & 5382400 & 3 \\
$370=2.5 .37$ & 1 & -1 & 3,1 & $(2,2)$ & $(6,2)$ & $(6,2,2)$ & 8761600 & 3 \\
$754=2.13 .29$ & 1 & 1 & 3,1 & $(2,2)$ & $(10,2)$ & $(10,2,2)$ & 36385024 & 3 \\
$3922=2.53 .37$ & 1 & 1 & 4,1 & $(2,2)$ & $(10,2)$ & $(10,2,2)$ & 984453376 & 3 \\
$4610=2.461 .5$ & 2 & 1 & 2,4 & $(2,2)$ & $(26,2)$ & $(26,2,2)$ & 1360134400 & 3 \\
$5122=2.197 .13$ & 1 & -1 & 5,1 & $(2,2)$ & $(14,2)$ & $(14,2,2)$ & 1679032576 & 3 \\
$5410=2.5 .541$ & 1 & 1 & 2,3 & $(2,2)$ & $(22,2)$ & $(22,2,2)$ & 1873158400 & 3 \\
\hline
\end{tabular}

Table 7: Invariants of $\mathbb{K}_{j}$ for the case $\left(\frac{p_{1}}{p_{2}}\right)=1$ and $\left(\frac{\pi_{1}}{\pi_{3}}\right)=-1$

\begin{tabular}{|l|l|l|l|l|l|l|l|l|l|}
\hline $2 \cdot p_{1} \cdot p_{2}$ & $q$ & $m, n$ & $\mathbf{C} l\left(\mathbb{K}_{1}\right)$ & $\mathbf{C} l\left(\mathbb{K}_{2}\right)$ & $\mathbf{C} l\left(\mathbb{K}_{3}\right)$ & $\mathbf{C} l\left(\mathbb{K}_{4}\right)$ & $\mathbf{C} l\left(\mathbb{K}_{5}\right)$ & $\mathbf{C} l\left(\mathbb{K}_{6}\right)$ & $\mathbf{C} l\left(\mathbb{K}_{7}\right)$ \\
\hline 2.5 .269 & 1 & 3,1 & $(30,10,2)$ & $(330,2,2)$ & $(120,12)$ & $(30,2,2)$ & $(30,2,2)$ & $(30,2,2)$ & $(30,2,2)$ \\
2.53 .29 & 1 & 3,1 & $(78,2,2)$ & $(78,6,2)$ & $(104,4)$ & $(130,2,2)$ & $(26,2,2)$ & $(26,2,2)$ & $(130,2,2)$ \\
2.5 .389 & 1 & 3,1 & $(42,14,2)$ & $(462,2,2)$ & $(168,4)$ & $(42,2,2)$ & $(42,6,2)$ & $(42,6,2)$ & $(42,2,2)$ \\
2.53 .37 & 1 & 4,1 & $(30,10,2)$ & $(30,2,2)$ & $(80,4)$ & $(10,2,2)$ & $(30,2,2)$ & $(30,2,2)$ & $(10,2,2)$ \\
2.13 .157 & 1 & 4,1 & $(26,26,2)$ & $(78,6,2)$ & $(208,4)$ & $(26,2,2)$ & $(26,2,2)$ & $(26,2,2)$ & $(26,2,2)$ \\
2.13 .269 & 1 & 4,1 & $(90,10,2)$ & $(990,6,2)$ & $(720,4)$ & $(90,2,2)$ & $(90,2,2)$ & $(90,2,2)$ & $(90,2,2)$ \\
\hline
\end{tabular}

Table 8: Invariants of $\mathbb{K}_{j}$ for the case $\left(\frac{p_{1}}{p_{2}}\right)=1$ and $\left(\frac{\pi_{1}}{\pi_{3}}\right)=1$

\begin{tabular}{|c|c|c|c|c|c|c|c|c|c|}
\hline$d=2 . p_{1} \cdot p_{2}$ & $q$ & $m, n$ & $\mathbf{C} l\left(\mathbb{K}_{1}\right)$ & $\mathbf{C} l\left(\mathbb{K}_{2}\right)$ & $\mathbf{C} l\left(\mathbb{K}_{3}\right)$ & $\mathbf{C l}\left(\mathbb{K}_{4}\right)$ & $\mathbf{C l} l\left(\mathbb{K}_{5}\right)$ & $\mathbf{C} l\left(\mathbb{K}_{6}\right)$ & $\mathbf{C} l\left(\mathbb{K}_{7}\right)$ \\
\hline $4498=2.13 .173$ & 1 & 2,2 & $(210,2,2)$ & $(42,14,2)$ & $(280,4)$ & $(28,2)$ & $(28,2)$ & $(28,2)$ & $(28,2)$ \\
$4610=2.461 .5$ & 2 & 2,4 & $(390,2,2)$ & $(234,2,2)$ & $(2496,4)$ & $(780,2)$ & $(260,2)$ & $(260,2)$ & $(780,2)$ \\
$5090=2.5 .509$ & 2 & 2,2 & $(234,2,2)$ & $(390,2,2)$ & $(624,4)$ & $(52,2)$ & $(156,2)$ & $(156,2)$ & $(52,2)$ \\
$5410=2.541 .5$ & 1 & 2,3 & $(110,2,2)$ & $(22,22,2)$ & $(1584,4)$ & $(44,2)$ & $(44,2)$ & $(44,2)$ & $(44,2)$ \\
$6322=2.29 .109$ & 1 & 2,2 & $(90,6,2)$ & $(18,6,2)$ & $(504,4)$ & $(180,2)$ & $(36,6)$ & $(36,6)$ & $(180,2)$ \\
$7090=2.709 .5$ & 2 & 2,2 & $(130,2,2)$ & $(442,2,2)$ & $(1872,4)$ & $(52,2)$ & $(52,2)$ & $(52,2)$ & $(52,2)$ \\
\hline
\end{tabular}


Table 9: Invariants of $\mathbb{L}_{j}$ for $\left(\frac{p_{1}}{p_{2}}\right)=1$ and $\left(\frac{\pi_{1}}{\pi_{3}}\right)=1, m$ is always 2

\begin{tabular}{|ccccccccccc|}
\hline$d$ & $q$ & $b$ & $n$ & $\mathbf{C} l\left(\mathbb{L}_{1}\right)$ & $\mathbf{C} l\left(\mathbb{L}_{2}\right)$ & $\mathbf{C} l\left(\mathbb{L}_{3}\right)$ & $\mathbf{C} l\left(\mathbb{L}_{4}\right)$ & $\mathbf{C} l\left(\mathbb{L}_{5}\right)$ & $\mathbf{C} l\left(\mathbb{L}_{6}\right)$ & $\mathbf{C} l\left(\mathbb{L}_{7}\right)$ \\
\hline 17090 & 2 & -1 & 2 & $(9240,420)$ & $(4620,2)$ & $(4620,2)$ & $(420,42)$ & $(420,42)$ & $(1680,30)$ & $(1680,10)$ \\
1586 & 1 & 1 & 2 & $(660,12)$ & $(220,2)$ & $(132,6)$ & $(132,6)$ & $(132,6)$ & $(88,2)$ & $(88,2)$ \\
2290 & 1 & -1 & 2 & $(780,60)$ & $(260,2)$ & $(60,10)$ & $(60,10)$ & $(60,10)$ & $(120,2)$ & $(120,2)$ \\
2626 & 2 & 1 & 2 & $(504,12)$ & $(252,6)$ & $(252,6)$ & $(36,6)$ & $(36,6)$ & $(144,6)$ & $(144,6)$ \\
4610 & 2 & 1 & 4 & $(18720,12)$ & $(2340,30)$ & $(2340,30)$ & $(780,30)$ & $(780,30)$ & $(12480,30)$ & $(12480,10)$ \\
5410 & 1 & -1 & 3 & $(3960,44)$ & $(44,22)$ & $(44,22)$ & $(220,2)$ & $(220,2)$ & $(1584,2)$ & $(1584,2)$ \\
\hline
\end{tabular}

Table 10: Invariants of $\mathbb{L}_{j}$ for $\left(\frac{p_{1}}{p_{2}}\right)=1$ and $\left(\frac{\pi_{1}}{\pi_{3}}\right)=-1$

\begin{tabular}{|cccccccccc|}
\hline$d$ & $b$ & $m, n$ & $\mathbf{C} l\left(\mathbb{L}_{1}\right)$ & $\mathbf{C} l\left(\mathbb{L}_{2}\right)$ & $\mathbf{C} l\left(\mathbb{L}_{3}\right)$ & $\mathbf{C} l\left(\mathbb{L}_{4}\right)$ & $\mathbf{C} l\left(\mathbb{L}_{5}\right)$ & $\mathbf{C} l\left(\mathbb{L}_{6}\right)$ & $\mathbf{C} l\left(\mathbb{L}_{7}\right)$ \\
\hline 1090 & 1 & 4,1 & $(240,6)$ & $(30,6,2)$ & $(6,6,6)$ & $(6,6,6)$ & $(6,6,6)$ & $(24,12)$ & $(48,6)$ \\
1490 & -1 & 3,1 & $(504,6)$ & $(126,6,2)$ & $(126,6,2)$ & $(18,6,6)$ & $(18,6,6)$ & $(72,2)$ & $(36,12)$ \\
4082 & -1 & 4,1 & $(624,78)$ & $(26,26,2)$ & $(78,6,2)$ & $(78,6,2)$ & $(78,6,2)$ & $(208,2)$ & $(104,4)$ \\
4706 & 1 & 3,1 & $(6120,6)$ & $(306,6,2)$ & $(510,6,2)$ & $(510,6,2)$ & $(510,6,2)$ & $(204,4)$ & $(408,2)$ \\
6994 & -1 & 4,1 & $(7920,30)$ & $(90,10,2)$ & $(990,6,2)$ & $(990,6,2)$ & $(990,6,2)$ & $(720,2)$ & $(360,4)$ \\
7474 & -1 & 5,1 & $(43680,2)$ & $(78,6,2)$ & $(2730,2,2)$ & $(2730,2,2)$ & $(2730,2,2)$ & $(1248,6)$ & $(208,4)$ \\
\hline
\end{tabular}

Table 11: Invariants of $\mathbb{K}_{j}$ for the case $\left(\frac{p_{1}}{p_{2}}\right)=-1$

\begin{tabular}{|c|c|c|c|c|c|c|c|c|c|c|}
\hline$d=2 \cdot p_{1} \cdot p_{2}$ & $q$ & $\pi$ & $m$ & $\mathbf{C} l\left(\mathbb{K}_{1}\right)$ & $\mathbf{C} l\left(\mathbb{K}_{2}\right)$ & $\mathbf{C} l\left(\mathbb{K}_{3}\right)$ & $\mathbf{C} l\left(\mathbb{K}_{4}\right)$ & $\mathbf{C l}\left(\mathbb{K}_{5}\right)$ & $\mathbf{C l}\left(\mathbb{K}_{6}\right)$ & $\mathbf{C l}\left(\mathbb{K}_{7}\right)$ \\
\hline $130=2.5 .13$ & 2 & -1 & 2 & $(12,2)$ & $(4,2)$ & $(8,4)$ & $(4,2)$ & $(2,2,2)$ & $(2,2,2)$ & $(4,2)$ \\
$370=2.37 .5$ & 1 & -1 & 3 & $(12,2)$ & $(60,2)$ & $(24,4)$ & $(12,2)$ & $(6,2,2)$ & $(6,2,2)$ & $(12,2)$ \\
$530=2.5 .53$ & 2 & 1 & 2 & $(84,2)$ & $(84,2)$ & $(56,4)$ & $(14,2,2)$ & $(28,2)$ & $(28,2)$ & $(14,2,2)$ \\
$1970=2.197 .5$ & 2 & 1 & 2 & $(260,2)$ & $(260,2)$ & $(312,12)$ & $(26,2,2)$ & $(52,2)$ & $(52,2)$ & $(26,2,2)$ \\
$2930=2.293 .5$ & 1 & 1 & 3 & $(252,2)$ & $(252,2)$ & $(56,4)$ & $(42,2,2)$ & $(28,2)$ & $(28,2)$ & $(42,2,2)$ \\
$3538=2.61 .29$ & 1 & -1 & 5 & $(84,2)$ & $(420,2)$ & $(224,4)$ & $(28,2)$ & $(14,2,2)$ & $(14,2,2)$ & $(28,2)$ \\
$5570=2.5 .557$ & 1 & -1 & 4 & $(660,2)$ & $(180,6)$ & $(240,4)$ & $(60,2)$ & $(30,2,2)$ & $(30,2,2)$ & $(60,2)$ \\
$6130=2.5 .613$ & 2 & -1 & 2 & $(1260,6)$ & $(180,2)$ & $(72,36)$ & $(36,2)$ & $(18,2,2)$ & $(18,2,2)$ & $(36,2)$ \\
\hline
\end{tabular}

Table 12: Invariants of $\mathbb{L}_{j}$ for the case $\left(\frac{p_{1}}{p_{2}}\right)=-1$

\begin{tabular}{|c|c|c|c|c|c|c|c|c|c|c|}
\hline$d=2 . p_{1} \cdot p_{2}$ & $q$ & $\pi$ & $m$ & $\mathbf{C} l\left(\mathbb{L}_{1}\right)$ & $\mathbf{C} l\left(\mathbb{L}_{2}\right)$ & $\mathbf{C} l\left(\mathbb{L}_{3}\right)$ & $\mathbf{C} l\left(\mathbb{L}_{4}\right)$ & $\mathbf{C} l\left(\mathbb{L}_{5}\right)$ & $\mathbf{C} l\left(\mathbb{L}_{6}\right)$ & $\mathbf{C} l\left(\mathbb{L}_{7}\right)$ \\
\hline $130=2.5 .13$ & 2 & -1 & 2 & $(24,2)$ & $(12,2)$ & $(12,2)$ & $(4,2)$ & $(4,2)$ & $(8,2)$ & $(4,4)$ \\
$370=2.5 .37$ & 1 & -1 & 3 & $(120,2)$ & $(60,2)$ & $(12,2)$ & $(12,2)$ & $(12,2)$ & $(24,2)$ & $(12,4)$ \\
$530=2.5 .53$ & 2 & 1 & 2 & $(168,6)$ & $(84,2)$ & $(84,2)$ & $(84,2)$ & $(84,2)$ & $(28,4)$ & $(56,2)$ \\
$1970=2.5 .197$ & 2 & 1 & 2 & $(1560,30)$ & $(260,2)$ & $(260,2)$ & $(260,2)$ & $(260,2)$ & $(156,12)$ & $(312,6)$ \\
$2930=2.293 .5$ & 1 & 1 & 3 & $(504,18)$ & $(252,6)$ & $(252,6)$ & $(252,6)$ & $(252,6)$ & $(84,12)$ & $(56,2)$ \\
$3538=2.29 .61$ & 1 & -1 & 5 & $(3360,6)$ & $(420,2)$ & $(420,2)$ & $(84,2)$ & $(84,2)$ & $(224,2)$ & $(112,4)$ \\
$5570=2.557 .5$ & 1 & -1 & 4 & $(7920,6)$ & $(180,6)$ & $(660,2)$ & $(660,2)$ & $(660,2)$ & $(240,2)$ & $(120,4)$ \\
$6130=2.5 .613$ & 2 & -1 & 2 & $(2520,90)$ & $(1260,6)$ & $(1260,6)$ & $(180,2)$ & $(180,2)$ & $(72,18)$ & $(36,36)$ \\
\hline
\end{tabular}

\section{REFERENCES}

[1] A. Azizi, Sur les unités de certains corps de nombres de degré 8 sur $\mathbb{Q}$, Ann. Sci. Math. Québec 29 (2005), no 2, 111-129. 
[2] A. Azizi, A. Zekhnini et M. Taous, On the unramified quadratic and biquadratic extensions of the field $\mathbb{Q}(\sqrt{d}, i)$, IJA, Volume 6 No. 24 (2012), 1169-1173.

[3] A. Azizi, A. Zekhnini et M. Taous, On the generators of the 2-class group of the field $\mathbf{k}=$ $\mathbb{Q}(\sqrt{d}, i)$, IJPAM, Volume 81 No. 5 (2012), 773-784.

[4] A. Azizi, A. Zekhnini et M. Taous, On the 2 -class field tower of $\mathbb{Q}\left(\sqrt{2 p_{1} p_{2}}, i\right)$ and the Galois group of its second Hilbert 2-class field, Collect. Math. DOI 10.1007/s13348-013-0085-4.

[5] A. Azizi et M. Taous, Détermination des corps $\mathbf{k}=\mathbb{Q}(\sqrt{d}, i)$ dont les 2-groupes de classes sont de type $(2,4)$ ou $(2,2,2)$, Rend. Istit. Mat. Univ. Trieste. 40 (2008), 93-116.

[6] A. Scholz, Über die Löbarkeit der Gleichung $t^{2}-D u^{2}=-4$,Math. Z. 39 (1934), 95-111.

[7] E. Benjamin, F. Lemmermeyer, C. Snyder, Real Quadratic Fields with Abelian 2-Class Field twoer, Journal of Number Theory, Volume 73, Number 2, December (1998), pp. 182-194 (13).

[8] E. Benjamin, F. Lemmermeyer, C. Snyder, Imaginary quadratic fields with $C l_{2}(k) \simeq(2 ; 2 ; 2)$, J. Number Theory 103 (2003), 38-70.

[9] F. Lemmermeyer, On 2-class field towers of imaginary quadratic number fields, Journal de Théorie des Nombres de Bordeaux 6 (1994), 261-272.

[10] F. Lemmermeyer, On 2-class field towers of some imaginary quadratic number fields, Abh. Math. Sem. Hamburg 67 (1997), 205-214.

[11] F. Lemmermeyer, Reciprocity Laws, Springer Monographs in Mathematics, Springer-Verlag. Berlin 2000.

[12] G.Gras, Class field theory, from theory to practice, Springer Verlag (2003).

[13] H. Kisilevsky, Number fields with class number congruent to 4 modulo 8 and Hilbert's Theorem 94, J. Number Theory 8 (1976), 271-279.

[14] J. P. Serre, Sur une question d'Olga Taussky, J. Number Theory 2 (1970), 235-236.

[15] O. Taussky, A Remark on the Class Field Tower, J. London Math. Soc. 12 (1937), 82-85.

[16] O. Taussky, A remark concerning Hilbert's Theorem 94, J. Reine Angew. Math. 239/240 (1970), 435-438.

[17] P. Kaplan, Sur le 2-groupe de classes d'idéaux des corps quadratiques, J. Reine angew. Math. 283/284 (1976), 313-363.

[18] The PARI Group, PARI/GP, Bordeaux, Version 2.4.4 (betta), May 9 2011, (http://pari.math.u-bordeaux.fr).

Abdelmalek Azizi and Abdelkader Zekhnini: Département de Mathématiques, FaCulté des Sciences, Université Mohammed 1, Oujda, Morocco

E-mail address: abdelmalekazizi@yahoo.fr

E-mail address: zekha1@yahoo.fr

Mohammed Taous: Département de Mathématiques, Faculté des Sciences et TechNiques, Université Moulay Ismail, Errachidia, Morocco

E-mail address: taousm@hotmail.com 